\title{
Reactive Oxygen Species Modulate Activity-Dependent AMPA Receptor Transport in C. elegans
}

\author{
${ }^{\circledR}$ Rachel L. Doser, ${ }^{\circledR}$ Gregory C. Amberg, and ${ }^{\circledR}$ Frederic J. Hoerndli \\ Department of Biomedical Science, Colorado State University, Fort Collins, Colorado 80523
}

The AMPA subtype of synaptic glutamate receptors (AMPARs) plays an essential role in cognition. Their function, numbers, and change at synapses during synaptic plasticity are tightly regulated by neuronal activity. Although we know that long-distance transport of AMPARs is essential for this regulation, we do not understand the associated regulatory mechanisms of it. Neuronal transmission is a metabolically demanding process in which ATP consumption and production are tightly coupled and regulated. Aerobic ATP synthesis unavoidably produces reactive oxygen species (ROS), such as hydrogen peroxide, which are known modulators of calcium signaling. Although a role for calcium signaling in AMPAR transport has been described, there is little understanding of the mechanisms involved and no known link to physiological ROS signaling. Here, using realtime in vivo imaging of AMPAR transport in the intact $C$. elegans nervous system, we demonstrate that long-distance synaptic AMPAR transport is bidirectionally regulated by calcium influx and activation of calcium/calmodulin-dependent protein kinase II. Quantification of in vivo calcium dynamics revealed that modest, physiological increases in ROS decrease calcium transients in C. elegans glutamatergic neurons. By combining genetic and pharmacological manipulation of ROS levels and calcium influx, we reveal a mechanism in which physiological increases in ROS cause a decrease in synaptic AMPAR transport and delivery by modulating activity-dependent calcium signaling. Together, our results identify a novel role for oxidant signaling in the regulation of synaptic AMPAR transport and delivery, which in turn could be critical for coupling the metabolic demands of neuronal activity with excitatory neurotransmission.

Key words: AMPAR; C. elegans; calcium signaling; ROS; synapses; transport

Significance Statement

Synaptic AMPARs are critical for excitatory synaptic transmission. The disruption of their synaptic localization and numbers is associated with numerous psychiatric, neurologic, and neurodegenerative conditions. However, very little is known about the regulatory mechanisms controlling transport and delivery of AMPAR to synapses. Here, we describe a novel physiological signaling mechanism in which ROS, such as hydrogen peroxide, modulate AMPAR transport by modifying activity-dependent calcium signaling. Our findings provide the first evidence in support of a mechanistic link between physiological ROS signaling, AMPAR transport, localization, and excitatory transmission. This is of fundamental and clinical significance since dysregulation of intracellular calcium and ROS signaling is implicated in aging and the pathogenesis of several neurodegenerative disorders, including Alzheimer's and Parkinson's disease.

Received Apr. 17, 2020; revised July 26, 2020; accepted Aug. 16, 2020.

Author contributions: R.L.D., G.C.A., and F.J.H. designed research; R.L.D. and F.J.H. performed research; R.L.D., G.C.A., and F.J.H. analyzed data; R.L.D. and F.J.H. wrote the first draft of the paper; R.L.D., G.C.A., and F.J.H. edited the paper; R.L.D. and F.J.H. wrote the paper.

The authors declare no competing financial interests.

F.J.H. was supported by the College of Veterinary Medicine and Biomedical Sciences and the Molecular, Cellular and Integrative Neuroscience Program at Colorado State University. G.C.A. was supported by R01 HD087347. Strains were obtained from the Caenorhabditis Genetics Center funded by National Institutes of Health Office of Research Infrastructure Programs P40 0D010440. We thank Dr. Attila Stetak (University of Basel, Switzerland) for providing GCaMP6f C. elegans reagents and Dr. Pulford (Colorado State University) for his technical assistance.

Correspondence should be addressed to Frederic J. Hoerndli at Frederic.Hoerndli@colostate.edu.

https://doi.org/10.1523/JNEUROSCI.0902-20.2020

Copyright $\odot 2020$ the authors

\section{Introduction}

The AMPA subtype of glutamate receptors (AMPARs) are essential for fast excitatory synaptic transmission (Ashby et al., 2008). The number of AMPARs at the synaptic surface is a key determinant of synaptic efficacy and is the result of a dynamic equilibrium between the number of receptors in intracellular pools and at the synaptic surface (Rosendale et al., 2003; Groc et al., 2009; Henley and Wilkinson, 2013). Although a few AMPARs can be synthesized locally (Hanus et al., 2016), the vast majority of AMPARs are synthesized in the neuronal soma, often far away from synapses, and are trafficked in a complex multistep process to dendrites and synapses (Hanus et al., 2016; Henley and Wilkinson, 2016; Brechet et al., 2017). Intracellular transport by molecular motors (C. H. Kim and Lisman, 2001; Setou et al., 
Table 1. Genetic alleles used, corresponding gene, effect of the mutation, and reported functional changes along with original references characterizing the allele

\begin{tabular}{|c|c|c|c|c|}
\hline$\overline{\text { Gene }}$ & Allele & Mutation & Functional change & References \\
\hline$g / r-1$ & ky176 & Premature stop & Truncated, unfunctional receptor & Maricq et al., 1995 \\
\hline \multirow[t]{2}{*}{ egl-19 } & $n 582(\mathrm{rf})$ & Missense mutation in $\$ 4$ domain & No calcium spikes & Trent et al., 1983; Liu et al., 2018 \\
\hline & $n 2368(g f)$ & Missense mutation in IS6 & $\begin{array}{l}\text { Delayed inactivation, prolonged } \\
\text { calcium spikes }\end{array}$ & $\begin{array}{l}\text { Lee et al., 1997; } \\
\quad \text { Laine et al., } 2014\end{array}$ \\
\hline \multirow[t]{2}{*}{ unc-43 } & $n 498 n 1186$ (If) & Premature stop & Protein null & $\begin{array}{l}\text { Park and Horvitz, 1986; Reiner et al., 1999; } \\
\text { Umemura et al., } 2005\end{array}$ \\
\hline & n498sd (gf) & $\begin{array}{l}\text { Missense mutation in } \\
\text { the active site }\end{array}$ & $\begin{array}{l}\text { Partial calcium-independent, } \\
\text { constitutive activation }\end{array}$ & \\
\hline$c t-2$ & ok1137 (If) & $\sim 1 \mathrm{~kb}$ deletion & Protein null & Spiró et al., 2012 \\
\hline $\operatorname{lin}-15$ & $n 765 t s$ & Frameshift mutation & Protein null & S. K. Kim and Horvitz, 1990 \\
\hline
\end{tabular}

2002; Hoerndli et al., 2013; Esteves da Silva et al., 2015; Hangen et al., 2018), exocytosis and endocytosis (Ehlers, 2000; Yudowski et al., 2007) as well as surface diffusion dynamics (Choquet and Triller, 2013) are all important steps of this complex trafficking. Intracellular AMPAR transport between different cellular pools of AMPARs is the least understood of these steps but is essential for synaptic transmission and plasticity (C. H. Kim and Lisman, 2001; Setou et al., 2002; Hoerndli et al., 2013). In addition, several studies have now shown that transcription of Kinesin-1 motors (Puthanveettil et al., 2008) and the number of AMPARs transported increase with neuronal activity (Hoerndli et al., 2015; Hangen et al., 2018). Furthermore, AMPAR exocytosis (Yudowski et al., 2007) and stopping during transport in dendrites also increase with activity (Hangen et al., 2018), suggesting that vesicle stops correlate with AMPAR delivery (Hoerndli et al., 2013). The correlation between stopping of vesicular cargo and delivery has also been observed for other postsynaptic cargoes (Guillaud et al., 2008; Ichinose et al., 2015; Stucchi et al., 2018; Bommel et al., 2019), but the regulation of stops and delivery of AMPARs are so far unknown.

Neuronal activity is associated with increased energy demands that is largely fulfilled by mitochondrial ATP production (Hall et al., 2012), which concurrently produces reactive oxygen species (ROS) (Halliwell, 1992). The main ROS species are hydrogen peroxide $\left(\mathrm{H}_{2} \mathrm{O}_{2}\right)$, the superoxide anion $\left(\mathrm{O}_{2}{ }^{-}\right)$, and the hydroxyl radical $\left(\mathrm{HO}^{-}\right)$(Halliwell, 1992). Previous studies have shown that ROS can affect calcium signaling mediated by NMDA glutamate receptors, voltage-gated calcium channels (VGCCs), and calcium release from the ER (Akaishi et al., 2004; Amberg et al., 2010; Todorovic and Jevtovic-Todorovic, 2014; Hidalgo and Arias-Cavieres, 2016). Interestingly, the effect of ROS varies widely depending on dosage, cell type, and model system used (Hidalgo and Arias-Cavieres, 2016; Wilson et al., 2018). This is illustrated by the fact that LTP is disrupted by elevated ROS (Bliss and Collingridge, 1993; Klann, 1998; Kamsler and Segal, 2003) as well as depletion of ROS (Kishida and Klann, 2007). Thus, the literature supports a link between ROS signaling and changes in neuronal excitability. However, whether this is because of ROS modulation of calcium signaling remains uncertain. In particular, there is a lack of direct evidence for the roles of physiological ROS on neuronal calcium signaling in vivo.

The transparent model Caenorhabditis elegans is well suited to study the effects of ROS on calcium signaling in neurons in vivo where circuits remain intact. More specifically, calcium and ROS sensors have been successfully used in vivo in C. elegans to study conserved signaling pathways (Sengupta and Samuel, 2009; Luo et al., 2014; Braeckman et al., 2016). In addition, C. elegans has been instrumental in uncovering translationally conserved calcium and ROS signaling mechanisms regulating neuronal activity, organismal aging, and neurodegeneration (Petriv and Rachubinski, 2004; Treusch et al., 2011; Back et al., 2012; Tardiff et al., 2013; Griffin et al., 2019; Zullo et al., 2019; Alvarez et al., 2020).

In this study, we start to address the possible link between ROS production and regulation of AMPAR transport using $C$. elegans. Single-neuron expression of SEP::mCherry::GLR-1 (the C. elegans homolog of the AMPAR subunit GluA1 tagged at the $\mathrm{N}$-terminus with SEP or Super Ecliptic Phluorine, a pH-sensitive form of GFP, and mCherry) and the calcium sensor GCAMP6f enabled us to quantify and characterize GLR-1 transport as well as changes in cytoplasmic calcium in vivo, in real time. Together with genetic and pharmacological manipulation of VGCC activity and ROS levels, our results show that AMPAR transport is directly regulated by activity-dependent calcium signaling. We also find that physiological increases in ROS levels decrease calcium and, as a result, AMPAR transport, delivery, and exocytosis. We further show that the targets of increased ROS are specific and involve L-type VGCC-dependent calcium signaling upstream of CaMKII activation. Together, our results suggest a mechanism by which physiological ROS signaling acts as a negative feedback mechanism regulating excitatory glutamatergic transmission by decreasing activity-dependent calcium influx and subsequent AMPAR transport.

\section{Materials and Methods}

Strains. C. elegans strains were maintained on nematode growth media and fed with the Escherichia coli strain OP50 at $20^{\circ} \mathrm{C}$ (Brenner, 2003). All animals used in experiments were hermaphrodites and the strains used in these experiments contained alleles listed in Table 1.

To visualize GLR-1, we used the integrated array akIs201 containing Prig-3::SEP::mCherry::GLR-1 (Hoerndli et al., 2015) to express dualtagged GLR-1 in the AVA glutamatergic interneurons. Transgenic strains were created by microinjection of lin-15(n765ts) worms with plasmids containing lin-15(+) to allow for phenotypic rescue of transgenic strains. The GCaMP6f-containing plasmid (Prig-3::GCaMP6f:: unc-54) was a gift from Attila Stetak and was used to create the strain csfEx62 [Prig-3::GCaMP6f::unc-54].

Confocal microscopy. Imaging was conducted on a spinning disk confocal microscope (Olympus IX83) equipped with 488 and $561 \mathrm{~nm} \mathrm{ex-}$ citation lasers (Andor ILE Laser Combiner). Images were captured using an Andor iXon Ultra EMCCD camera through either a $10 \times / 0.40$ or a $100 \times / 1.40$ oil objective (Olympus). Devices were controlled remotely for image acquisition using MetaMorph 7.10.1 (Molecular Devices).

Transport imaging and analysis. All transport imaging was conducted on strains containing akIs201 in the glr-1 null background (ky176). 1-day-old adults from these strains were mounted on a $10 \%$ agarose pad with $1.6 \mu \mathrm{l}$ of a mixture containing equal measures of polystyrene beads (Polybead, catalog \#00876-15, Polysciences) and $30 \mathrm{~mm}$ muscimol (catalog \#195336, MP Biomedicals). The worm was positioned to place the AVA interneurons in close proximity to the coverslip 
through which the AVA neurites would be imaged. Once the neurons were located using the $100 \times$ objective and a $561 \mathrm{~nm}$ excitation laser, a proximal section of the neurites was photobleached using a $3 \mathrm{~W}, 488 \mathrm{~nm}$ Coherent solid-state laser (Genesis MX MTM) set to $0.5 \mathrm{~W}$ output and a $1 \mathrm{~s}$ pulse time. The photobleaching laser was targeted to a defined portion of AVA using a Mosaic II digital mirror device (Andor Mosaic 3) controlled through MetaMorph. Immediately following photobleaching, a 500 -frame image stream was collected in a single $z$ plane with the $561 \mathrm{~nm}$ excitation laser and a $100 \mathrm{~ms}$ exposure time. Kymographs were generated using the Kymograph tool in MetaMorph with a 20 pixel line width as previously reported (Hoerndli et al., 2013). Transport quantification was done blinded to the genotype and condition by manually counting all transport events from resultant kymographs. Transport stops and velocities, on the other hand, were analyzed unblinded by manually tracing $\sim 10$ transport events per kymograph using the ImageJ plugin KymoAnalyzer (Neumann et al., 2017). Individual traces were selected for inclusion in this analysis if the fluorescence and image focus allowed for the event to be readily traced throughout the entirety of the kymograph (50 s). At least 10 kymographs per experimental group were used for the stop and velocity analyses.

Fluorescence recovery after photobleaching (FRAP). Strains containing akIs201 were mounted for imaging as described above. First, an appropriate proximal region of AVA was localized and memorized using MetaMorph's stage position memory function. Second, an image stack was acquired using the $561 \mathrm{~nm}$, then the $488 \mathrm{~nm}$ excitation laser $(500 \mathrm{~ms}$ exposure) around the AVA process (20 images were taken every $0.25 \mu \mathrm{m}$, starting $2.5 \mu \mathrm{m}$ below to $2.5 \mu \mathrm{m}$ above the process, which required $\sim 30 \mathrm{~s}$ total imaging time). Third, $\sim 80-\mu \mathrm{m}$-long sections of AVA proximal and distal to the imaging region were photobleached using the same photobleaching settings as previously described. The imaging region was photobleached; and immediately after, two image stacks (with $561 \mathrm{~nm}$, then $488 \mathrm{~nm}$ excitation) were acquired for the 0 min time point. This was repeated at 2, 4, 8, and 16 min following the photobleaching of the imaging region. Finally, image stacks from all time points were converted to maximum projections using MetaMorph's stack arithmetic function. The average fluorescence in the imaging region at each time point was analyzed using the region measurement tool in ImageJ 1.51s (Java 1.8). The background fluorescence (i.e., outside of the AVA) from each maximum projection was then subtracted from the average fluorescence of the imaging region. The resulting fluorescence from the maximum projections immediately following photobleaching $(0 \mathrm{~min})$ was subtracted from the fluorescence values of all subsequent time points. These values were then divided by the average fluorescence of the neurite before photobleaching to determine the percent of signal recovery for each channel within the imaging region.

In vivo calcium imaging. All strains used for calcium imaging experiments contained the extrachromosomal array csfEx62 expressing GCAMP6f in the AVA interneurons in the lin-15(n765ts) genetic background. Eight to 101 -day-old adult animals with the array were selected and placed on a $10 \%$ agar pad with $2 \mu$ of standard M9 buffer (common C. elegans culturing buffer) (Stiernagle, 2006). C. elegans were thus constrained but not immobilized similar to when placed in a microfluidics chamber (Chronis et al., 2007). Animals were imaged using the $10 \times$ objective on the spinning disk confocal. A $60 \mathrm{~s}$ image stream consisting of 240 images with a $250 \mathrm{~ms}$ exposure time was acquired using the $488 \mathrm{~nm}$ excitation laser. During imaging, animals spontaneously attempt reversals, which is correlated with activation of the AVA, thus increasing cytoplasmic calcium (Ben Arous et al., 2010) and resulting in changes in GCaMP6f fluorescence in our strains. We report the total activity or total cytoplasmic calcium during each $60 \mathrm{~s}$ stream (see Fig. $2 A, B$ ). This was calculated using the following approach. For each frame, the maximum fluorescence $\left(\mathrm{F}_{(\mathrm{t})}\right)$ was quantified using MetaMorph's region measurement tool by manually defining the region of the image stream containing the AVA cell bodies. Attempts at reversals exhibited large calcium transients, whereas attempts at forward movement or absence of movement was correlated with only small variations considered as basal fluctuations. The baseline $\left(\mathrm{F}_{\mathrm{min}}\right)$ was defined as the average GCAMP6f signal when worms are immobile or during forward movement as this was previously shown not to activate AVA (Ben Arous et al., 2010).
GCAMP6f signal during this time was observed to be within $30 \%$ of the overall minimum value of GCAMP6f. Maximum fluorescence values for each frame were imported into a customized Excel document containing modules created with Excel's visual basic editor. One module calculates the average baseline $\left(\mathrm{F}_{\min }\right)$ by averaging all values within $30 \%$ of lowest value. This value was used to determine the $\Delta \mathrm{F}\left(\mathrm{F}_{(\mathrm{t})}-\mathrm{F}_{\min }\right)$ for each frame normalized to the average baseline $\left(\Delta \mathrm{F} / \mathrm{F}_{\mathrm{min}}\right)$ (Larsch et al., 2013). Total activity was defined as the sum of all $F_{(t)}$ values greater than $F_{\min }$ (green area below the curve of all peaks of the $60 \mathrm{~s}$ recording; see Fig. $2 B$ ) normalized to the average baseline (average of the values below dotted line of the 60 s recording; see Fig. $2 B$ ).

Spontaneous reversal quantification. The spontaneous reversal rate (reversal/min) was quantified using the semiautomated tracking system of the WormLab System (MBF Bioscience). Briefly, for each trial, 5-8 1day-old adult worms were selected off plates with bacterial food and transferred on food-free plates twice with a rest of $\sim 2 \mathrm{~min}$ after each transfer. Spontaneous locomotion was then recorded for $1 \mathrm{~min}$ on the second plate and the video analyzed blinded to genotype. After detection of all animals and tracks by the semiautomatic tracking system, all tracks were manually verified and corrected, if necessary, still blind to genotype. N2, WT animal locomotion and reversal rates were consistent with previously reported values: $3.78 \pm 0.26 \mathrm{reversal} / \mathrm{minute}$ compared with reported $\sim 4 \pm 0.2$ (Monteiro et al., 2012).

Hydrogen peroxide and nemadipine treatments. Worms were acutely treated with $\mathrm{H}_{2} \mathrm{O}_{2}$ by placing the worm in $1.6 \mu \mathrm{l}$ of solution containing the appropriate concentration of $\mathrm{H}_{2} \mathrm{O}_{2}$ with either $15 \mathrm{~mm}$ muscimol and polystyrene beads (for transport imaging) or M9 buffer (for calcium imaging); $\sim 5 \mathrm{~min}$ following the beginning of the treatment, image streams were acquired. Physiologic intracellular concentrations of $\mathrm{H}_{2} \mathrm{O}_{2}$ range from 10 to $100 \mathrm{~nm}$ in vertebrate neurons (Sies, 2017), so 10, 50 and $100 \mathrm{~nm}$ was used for our experiments.

A 0.5 mm stock of nemadipine (VWR, catalog \#89151-228) was dissolved in $0.1 \%$ DMSO, and the concentration was adjusted to $10 \mu \mathrm{M}$ (a concentration reported to decrease calcium influx in exposed neurons in vivo by $\sim 70 \%$ ) (Larsch et al., 2013) with M9 buffer and OP50 liquid culture immediately before a $30 \mathrm{~min}$ treatment. During the treatments, 1day-old adult control worms were placed into $1.5 \mathrm{ml}$ Eppendorf tubes containing either control media or the pharmacological agent and placed on a rocker for oxygenation. The worms were then pipetted onto fresh nematode growth media/OP50 plates immediately before being moved to an agar pad for imaging.

Image presentation and data analysis. All images were acquired under nonsaturating conditions. Quantification of GLR-1 transport, FRAP, and calcium imaging is described in the appropriate sections above. Representative images shown were chosen and processed following analysis only to the extent necessary to appreciate the corresponding quantifications. Image processing (RGB colors, cropping, adjustment of brightness, and contrast) was performed in Photoshop (21.1.1). For FRAP images, the mCherry signal of SEP::mCherry::GLR-1 is shown in magenta for colorblind vision. This was achieved in Photoshop by duplicating the $561 \mathrm{~nm}$ information to create the red and blue channels of the RGB image, which was then merged to create the magenta image as published previously (Hoerndli et al., 2013). All images in each panel were identically processed.

Experimental design and statistical analysis. All experiments were performed using 1-day-old adult hermaphrodite C. elegans animals as determined by a single row of eggs and by picking as precisely identifiable L4 stage larva 18-30 h before imaging and behavior experiments. Each dataset contained at least three experimental replicates with 4 or 5 replicates needed for datasets containing more genotypes or conditions (see Figs. 5-7). The total number of animals analyzed is indicated in the figure legends. All mutant strains were back-crossed at least $2 \times$ with $\mathrm{N} 2$ WT animals. All imaging reagents, such as SEP::mCherry::GLR-1 and GCAMP6f, were crossed into strains carrying genetic mutations in the exact same way, verifying the presence of the KO glr-1 allele ky176 and genetic mutations using PCR genotyping on at least 2 generations. Primer sequences are available on request.

For statistical analysis, all datasets were screened for outliers using a Thompson Tau test. For datasets including only two experimental 
groups, statistical significance was tested using a two-tailed Student's $t$ test. For datasets comparing more than two experimental groups, a oneway Brown-Forsythe ANOVA with a Dunnett's correction for multiple comparisons was used. FRAP differences between groups were determined using an extra sum-of-squares $F$ test of the nonlinear hyperbolic regression fit to the data. All statistical analyses were performed using Prism 8 software. Statistical details, numbers of ROIs and animals analyzed, and $p$ values are indicated in detail in each section of Results. Data are presented as mean \pm SEM unless otherwise stated.

\section{Code/software}

Code for custom Excel modules used for analysis of calcium imaging is available on request.

\section{Results}

C. elegans is a useful model for studying long-distance AMPAR transport dynamics in vivo. Here we use a dual-tagged AMPAR subunit, SEP::mCherry::GLR-1 in the glr-1(ky176) genetic background, expressed in a single pair of glutamatergic neurons (AVA) to analyze how transport, delivery, and exocytosis of GLR-1 are modulated by cytoplasmic calcium and ROS. AVA are long, ventrally running unipolar interneurons with cell bodies in the head of the animal that express AMPA and NMDA subtypes of glutamate receptors (Maricq et al., 1995). To reveal dim GLR-1 transport events, we used a photobleaching approach combined with continuous imaging of the mCherry signal to visualize GLR-1 transport in the proximal region of AVA (Fig. 1A). Both anterograde (Fig. $1 A$, blue arrowheads) and retrograde (Fig. $1 A$, fuchsia arrowheads) transport events can be visualized as single particles advancing in opposite directions at different time points (Fig. $1 A$, time point images 1-3). The trajectories of these transport events can be visualized and analyzed in a kymograph representing their position on $x$ axis and time on the $y$ axis (Fig. $1 A$, bottom right). The total amount of GLR-1 transport as well as velocities and stopping of transport events can be quantified using these kymographs. In control animals, the number of transport events as well as the average anterograde velocity obtained in our hands (Fig. 1C,F) are similar to what we reported previously and reported for vertebrate AMPAR transport in hippocampal neurons (Ju et al., 2004; Hoerndli et al., 2015; Hangen et al., 2018).

\section{Activity-dependent calcium signaling regulates AMPAR transport}

Recently, we and others have shown that long-distance AMPAR transport is regulated dynamically by neuronal activity. Although studies have shown that CaMKII activation is required for activity-dependent AMPAR transport, the exact signaling pathways leading to CaMKII activation are still unclear (Hayashi et al., 2000; Hoerndli et al., 2015; Hangen et al., 2018). In C. elegans neurons, the majority of neuronal depolarization is achieved by VGCCs, specifically by L-type VGCC (L-VGCC), while voltage-gated sodium channels are absent (Serrano-Saiz et al., 2013). C. elegans animals expressing VGCCs with reduced calcium conductance have altered synaptic distribution (Rongo and Kaplan, 1999) and diminished transport of AMPARs (Hoerndli et al., 2015). A necessary next step in understanding the regulation of longdistance transport of AMPARs to and from synapses is to determine whether this process is directly regulated by increased cytoplasmic calcium leading to CaMKII activation. If this is the case, then we would expect transport characteristics, such as export from the soma as well as transport velocities and pausing, to correlate with activity-dependent changes in cellular calcium levels.

To test this hypothesis, we took a genetic approach using strains with a reduced- or gain-of-function mutation in egl-19, the sole L-VGCC gene in C. elegans, leading, respectively, to a decrease or increase in calcium influx (Liu et al., 2018). In the egl-19 reduced-function ( $\mathrm{rf}$ ) mutant, GLR-1 transport out of the cell body was significantly decreased $(10.2 \pm 1.2$, mean \pm SEM, transport events per kymograph, $n=16, p=0.0036$; Fig. $1 B, C)$ compared with controls (17.9 \pm 1.7 events, $n=19)$. Conversely, in the egl-19 gain-of-function (gf) mutant, GLR-1 transport was upregulated ( $26.7 \pm 1.7$ events, $n=17, p=0.0037$; Fig. $1 B, C$ ). To ensure that these changes in transport are indeed because of altered calcium influx, we acutely treated animals containing SEP::mCherry::GLR-1 in the $g l r-1(k y 176)$ background with the L-type-specific VGCC blocker, nemadipine (Kwok et al., 2006). A 30 min pretreatment with $10 \mu \mathrm{M}$ nemadipine caused a decrease in total transport $(12.0 \pm 1.5$ events, $n=14$, compared with $23.4 \pm 2.5$ events in untreated controls, $n=12, p=0.0005$; Fig. $1 D, E)$ similar to egl-19(rf) $(10.2 \pm 1.2$; Fig. $1 C)$. Both nemadipine treatment and egl-19(rf) showed a similar reduction in calcium in AVA as measured by GCAMP6f (data not shown). These results indicate that not only is calcium through VGCCs required (Hoerndli et al., 2015), but it also directly and bidirectionally regulates AMPAR transport to and from the cell body.

To better understand how AMPAR transport dynamics are impacted by calcium, we quantified the velocity and stop frequency of individual GLR-1-containing vesicles in the egl-19 mutants. We found that the increased calcium in egl-19(gf) mutants results in a slightly faster anterograde instantaneous velocity $(1.37 \pm 0.03 \mu \mathrm{m} / \mathrm{s}, n=87$ events, $p=0.003)$ compared with control animals $(1.23 \pm 0.02 \mu \mathrm{m} / \mathrm{s}, n=90$; Fig. $1 F)$. The distribution frequency of the anterograde velocities in each group revealed that the increase in egl-19(gf) is because of more vesicles traveling at higher speeds $(1.6-2.0 \mu \mathrm{m} / \mathrm{s}$; Fig. $1 G)$. Retrograde transport velocities, however, were not significantly changed in the egl-19(gf) mutants (Fig. $1 F, H)$. In addition, we observed a significant increase in the percent time spent stopped for vesicles moving in either direction in egl-19(gf) (29.9 $\pm 2.7 \%)$ compared with controls $(20.9 \pm 1.8 \%, p=0.043, n>95$; Fig. $1 I)$. Decreased calcium in egl-19(rf) mutants decreases the instantaneous velocity of anterograde GLR-1 transport $(1.08 \pm 1.1 \mu \mathrm{m} / \mathrm{s}, n=50$, $p=0.0037$ ), but surprisingly had the opposite effect on instantaneous velocity of retrograde transport $(1.4 \pm 0.06 \mu \mathrm{m} / \mathrm{s}, n=59$, compared with $0.9 \pm 0.06 \mu \mathrm{m} / \mathrm{s}$ in controls, $n=44, p<0.0001$; Fig. $1 F)$. The percent time vesicles moving in either direction spent stopped was also drastically decreased in egl-19(rf) mutants (paused $10.5 \pm 1.5 \%$ of the time, $p=0.0011, n=79$; Fig. $1 I$ ).

Cytoplasmic calcium increases from L-VGCC and other sources are known to activate CaMKII (Bayer and Schulman, 2019), which in turn has been shown to be required for normal AMPAR transport (Hoerndli et al., 2015; Hangen et al., 2018). We sought to determine whether and to what degree CaMKII activation regulates AMPAR transport. For these experiments, we used strains harboring genetic loss- and gain-of-function mutations of UNC-43, the sole C. elegans ortholog of CaMKII. The unc-43 loss-of-function (lf) mutation leads to a complete loss of UNC-43 (Reiner et al., 1999), whereas the gain-of-function (gf) allele causes partial calcium-independent, constitutive activation of UNC-43 (Umemura et al., 2005). Animals with unc-43(lf) showed a drastic decrease in GLR-1 transport $(0.78 \pm 0.30, n=14, p<0.0001)$, whereas animals with unc-43 (gf) showed a dramatic increase in GLR-1 transport $(36.3 \pm 2.04$ 
A

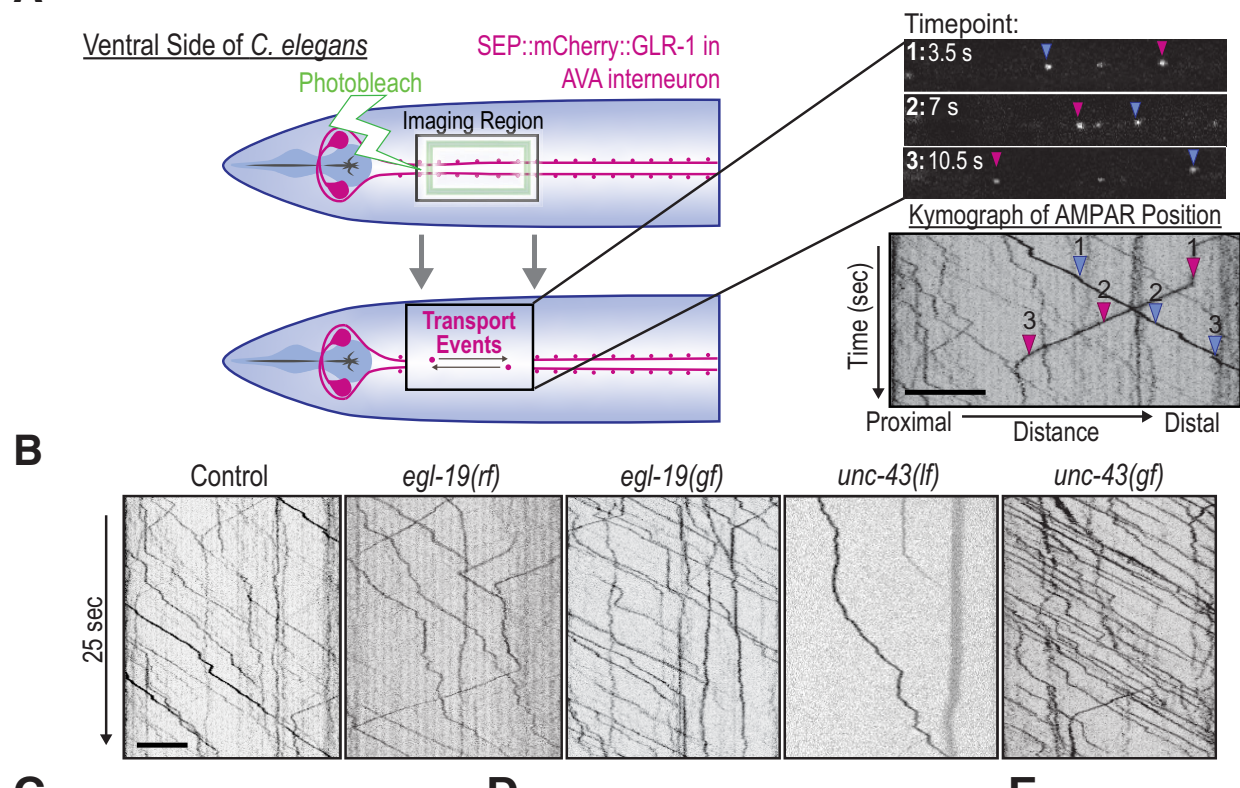

C

$\mathbf{F}$
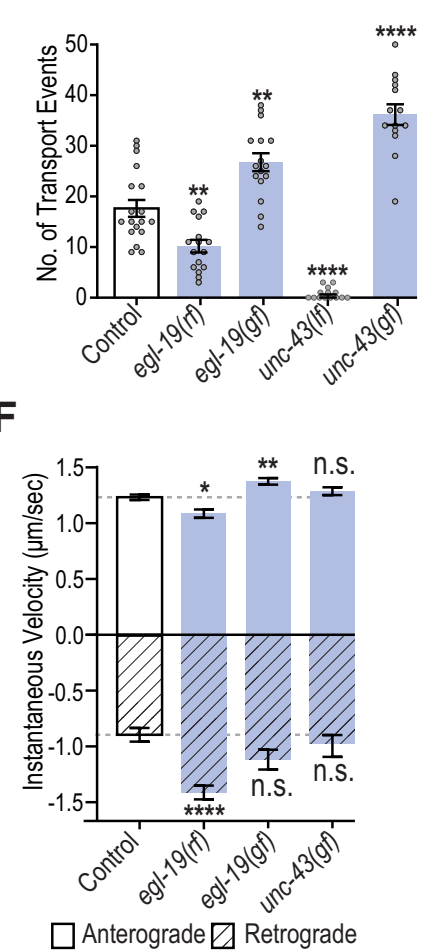

D

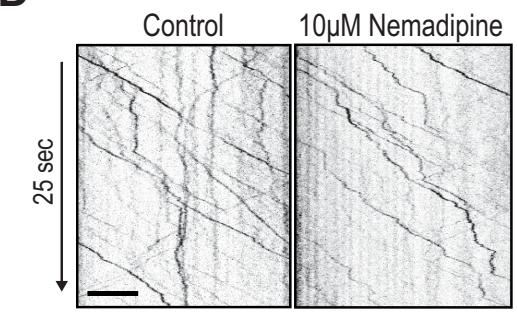

G

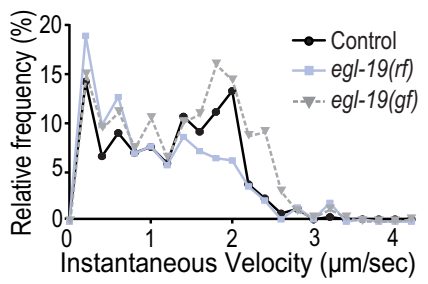

H

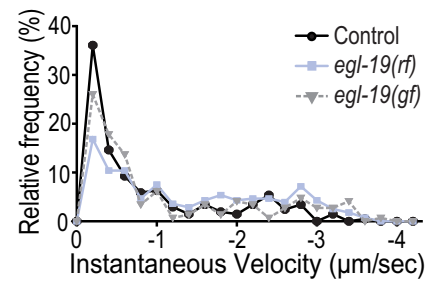

E

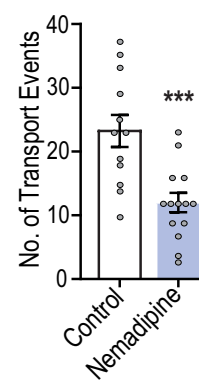

Figure 1. Activity-dependent calcium signaling regulates GLR-1 transport. A, Left, Diagram illustrating the location and procedure for in vivo imaging of mCherry::SEP::GLR-1 in AVA. Top right, Representative images at three time points showing positions of AMPAR-containing vesicles as they progress away from the cell body (blue arrowhead) and toward the cell body (fuchsia arrowhead). Bottom right, Kymograph displaying the position ( $x$ axis) of AMPAR-containing vesicles over time ( $y$ axis). Arrowheads indicate position of vesicles in kymograph that correspond to representative images at time points 1-3. Scale bar, $5 \mu \mathrm{m}$. All animals are expressing SEP:::mCherry::GLR-1 in the glr-1(ky176) background. For all transport experiments, animals with no additional mutations in this background are referred to as "controls." B, $25 \mathrm{~s}$ of representative kymographs from controls, egl-19 reduced-function (rf), egl-19 gain-of-function (gf), unc-43 loss-offunction (If), and unc-43 gain-of-function (gf) mutants. Scale bar, $5 \mu \mathrm{m}$. C, Total GLR-1 transport (anterograde and retrograde events) quantified from kymographs representative of a $50 \mathrm{~s}$ image stream ( $n>14$ worms for each group). ${ }^{* *} p=0.036,{ }^{* * * *} p<0.0001$, compared with controls. $\boldsymbol{D}, 25 \mathrm{~s}$ of representative kymographs of DMSO (control) and $10 \mu \mathrm{m}$ nemadipine-treated worms. Scale bar, $5 \mu \mathrm{m}$. $\boldsymbol{E}$, Total GLR-1 transport from DMSO controls $(n=12)$ and nemadipine-treated groups $(n=14)$. ${ }^{* * *} p=0.005$, compared with DMSO control. $\boldsymbol{F}-\mathbf{I}$, Stop and velocity analysis of $>60$ transport events from controls, egl-19(rf), egl-19(gf), and unc-43(gf) mutants. $\boldsymbol{F}$, Instantaneous velocity of AMPAR vesicles traveling in either an anterograde (solid) or retrograde fashion (diagonal lines; $n>60$ transport events). n.s. $=$ not significant, ${ }^{*} p=0.012,{ }^{* *} p=0.003$, ${ }^{* * * *} p<0.0001$, compared with controls. $\boldsymbol{G}, \boldsymbol{H}$, Distribution of instantaneous velocities (binned every $0.2 \mu \mathrm{m} / \mathrm{s}$ ) for anterograde $(\boldsymbol{G})$ and retrograde $(\boldsymbol{H})$ transport for controls, egl-19(rf), and egl-19(gf) mutants. $\boldsymbol{I}$, The percent of time GLR-1-containing vesicles spent stopped in each genotype as quantified from the same transport events in $\boldsymbol{A} .{ }^{*} p=0.043$, ${ }^{* *} p=0.001$, ${ }^{* * * *} p<0.0001$, compared with controls. Error bars indicate SEM. 

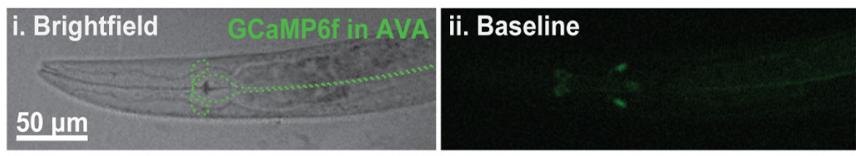

C
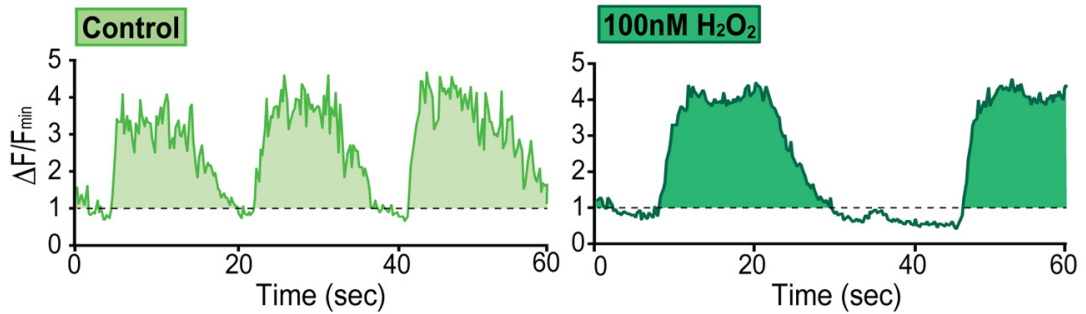

$\mathbf{F}$
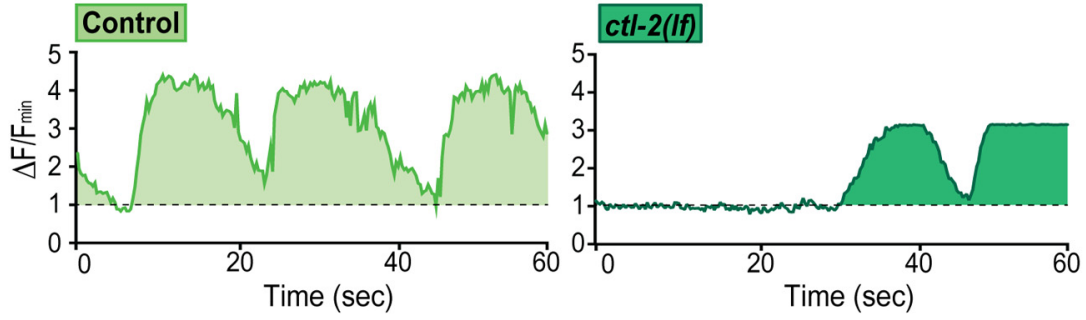

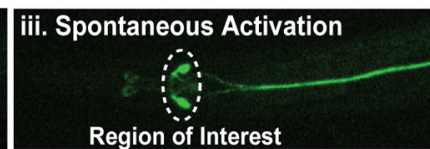

D

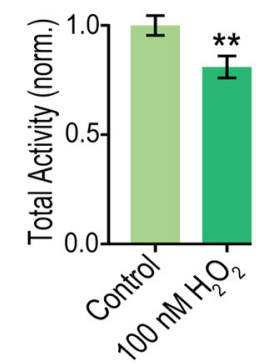

G

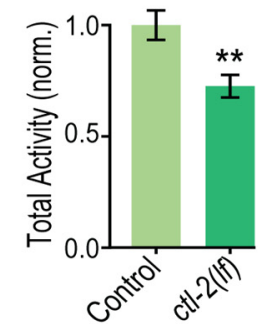

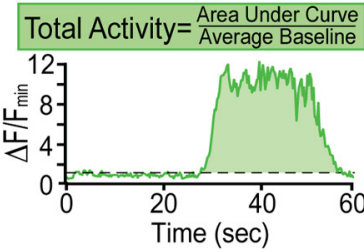

E

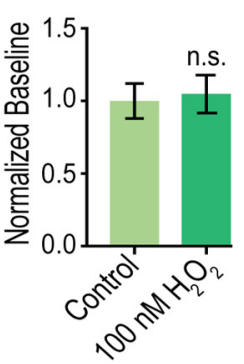

H

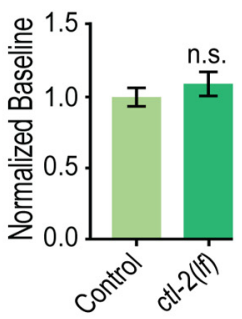

Figure 2. Physiologic increase in ROS leads to decreased activity-dependent fluctuations in somatic calcium. $\boldsymbol{A}$, Brightfield and fluorescent confocal images of the ventral side of a $\boldsymbol{C}$. elegansexpressing GCaMP6f in the AVA interneuron. Scale bar, $50 \mu \mathrm{m}$. B, GCaMP6f $\Delta \mathrm{F} / \mathrm{F}_{\min }$ over time. Gray dashed line indicates baseline threshold (30\% of minimum fluorescence value). Fluorescence values above that threshold are summed (area under the curve for all peaks) and normalized to the average baseline value ( $F_{\text {min }}$ ) to calculate total activity during the $60 \mathrm{~s}$ recording. For all GCaMP experiments, all groups express GCaMP6f in AVA and are referred to as "control" if no additional $\mathrm{H}_{2} \mathrm{O}_{2}$ treatment or mutation was added. $\mathrm{C}$, Representative traces of somatic GCaMP6f fluorescence in AVA interneurons for $60 \mathrm{~s}$ normalized to baseline fluorescence $\left(\Delta \mathrm{F} / \mathrm{F}_{\min }\right)$ from control $(n=56)$ and $100 \mathrm{~nm} \mathrm{H}_{2} \mathrm{O}_{2}$-treated $(n=58)$ animals. Dashed line indicates $\mathrm{F}_{\text {min. }}$. D. Total activity of GCaMP normalized to untreated controls. ${ }^{* *} p=0.006$. $\boldsymbol{E}$, Average fluorescence baseline of controls and $\mathrm{H}_{2} \mathrm{O}_{2}$-treated animals normalized to control group ( $n \geq 50$ ), n.s. $=$ not significant. $\boldsymbol{F}$, Representative traces of somatic GCaMP6f fluorescence in AVA interneurons for $60 \mathrm{~s}$, normalized to baseline fluorescence in control ( $n=50)$ and catalase mutants ( $c t l-2(I f)$, $n=51$ ). Dashed line indicates $F_{\min }$. $\boldsymbol{G}$, Total activity normalized to controls. ${ }^{* *} p=0.001$. $\boldsymbol{H}$, Average fluorescence baseline of controls and catalase mutants normalized to controls ( $n \geq 50$ ), n.s. $=$ not significant.

events, $n=14, p<0.0001$; Fig. $1 B, C)$ compared with controls. Interestingly, in $u n c-43(g f)$ mutants, instantaneous transport velocity was unchanged (Fig. $1 F$ ), but the percentage of time all transport vesicles spent stopped was drastically decreased to nearly $0 \%$ (vesicles stopped $0.06 \pm 0.49 \%$ of the time, $n=58$, compared with $20.9 \pm 1.8 \%$ in controls, $n=95, p<0.0001$; Fig. $1 I$ ). We were unable to quantify velocities and stop frequency of GLR-1 transport in unc-43(lf) because of the low numbers of transport events per kymograph (less than one per kymograph on average).

Collectively, these data demonstrate that calcium influx and calcium signaling by CaMKII directly regulate GLR-1 transport, with differential regulation of the dynamics (velocity and stops) of anterograde and retrograde transport events. These findings advance our understanding of AMPAR transport by delineating a mechanism in which neuronal activity upregulates and downregulates the quantity and dynamics of AMPAR transport.

Increased ROS levels within physiological range modulate activity-dependent changes in cytoplasmic calcium

A growing field of evidence shows that calcium-dependent signaling is modified or coregulated by ROS (Görlach et al., 2015). Additionally, several studies have shown that neural excitability and synaptic plasticity are modified by ROS signaling (Yermolaieva et al., 2000; Kishida and Klann, 2007). More recently, a few studies have shown that function of VGCCs, including the L-type, is altered by increases in ROS above physiological concentrations (Todorovic and Jevtovic-Todorovic, 2014; Dang et al., 2018). However, we have a poor understanding of if and how physiological ROS signaling impacts activity-dependent fluctuations in neuronal calcium levels. Given this gap in knowledge and the growing interest in how calcium and ROS signaling act to regulate neuronal excitation, we tested whether slight perturbations of ROS produce observable changes in cytoplasmic calcium levels and the resultant signaling. First, using cell-specific expression of the genetically encoded, fluorescent calcium indicator GCaMP6f (Akerboom et al., 2013), we were able to visualize calcium transients in vivo in the soma of AVA (Fig. $2 A, B$ ). Using this technique, we observe temporal dynamics (i.e., transient frequency and duration) of calcium transients similar to previous reports (Larsch et al., 2013; Gordus et al., 2015).

To determine whether cytoplasmic calcium is impacted by $\mathrm{H}_{2} \mathrm{O}_{2}$, the most stable and common form of endogenous ROS in cells (Bienert et al., 2006), we subjected WT worms expressing GCaMP6f in the AVA to a 5 min pretreatment of $100 \mathrm{nM} \mathrm{H}_{2} \mathrm{O}_{2}$ before imaging. With this technique, we determined that acute treatment with a $\mathrm{H}_{2} \mathrm{O}_{2}$ concentration within the physiological range (10-100 nm) (Sies, 2017) decreased the total GCaMP 
activity to $80.9 \pm 0.07 \%$ of that of untreated controls $(n>55$ for each group, $p=0.006$; Fig. $2 C, D$ ). Furthermore, we saw no effect of $\mathrm{H}_{2} \mathrm{O}_{2}$ treatment on baseline fluorescence (Fig. $2 E$ ), indicating that the modest increase in ROS because of this treatment does not detectably change the fluorescence properties of GCaMP itself or basal calcium levels. We conclude that an acute increase in $\mathrm{H}_{2} \mathrm{O}_{2}$ decreases the total amount of cytoplasmic calcium over the recording time (60 s) without drastically modifying the amplitude of the changes in cytoplasmic calcium in the cell body of AVA.

We sought additional evidence by using a genetic strategy to increase intracellular $\mathrm{H}_{2} \mathrm{O}_{2}$. To this end, we obtained a strain harboring a loss-of-function mutation in the gene encoding the primary C. elegans catalase (ctl-2), which decomposes $\sim 80 \%$ of all intracellular $\mathrm{H}_{2} \mathrm{O}_{2}$ to water, including in neuronal tissue (Petriv and Rachubinski, 2004). In these catalase mutants, total GCaMP activity is significantly decreased (27.4 $\pm 0.08 \%$ lower, $p=0.001$; Fig. $2 F, G)$. Again, the baseline of GCaMP fluorescence in $c t l-2(l f)$ was not different from that in control animals (Fig. $2 \mathrm{H}$ ). These results support that both acute and chronic increases in $\mathrm{H}_{2} \mathrm{O}_{2}$ within the physiological range attenuate activity-dependent increases in cytoplasmic calcium in C. elegans neurons. Based on the identified regulatory role of calcium on GLR-1 transport, we then hypothesized that these same modest increases in ROS may affect GLR-1 transport and delivery to synapses.

\section{Physiologic ROS signaling regulates AMPAR transport and delivery to synapses}

To test whether ROS levels impact GLR-1 transport, we quantified GLR-1 transport as previously described following a $5 \mathrm{~min}$ pretreatment with 10,50 , or $100 \mathrm{nM} \mathrm{H}_{2} \mathrm{O}_{2}$ in which animals swam freely before imaging in the same $\mathrm{H}_{2} \mathrm{O}_{2}$-containing solution. We found that worms treated with $\mathrm{H}_{2} \mathrm{O}_{2}$ had significantly fewer AMPAR transport events ranging from $14.65 \pm 1.59$ events at $10 \mathrm{nM} \mathrm{H}_{2} \mathrm{O}_{2}$ to $11.35 \pm 1.14$ at $100 \mathrm{nM} \mathrm{H}_{2} \mathrm{O}_{2}$ (compared with $23.0 \pm 1.09$ events in untreated animals, $n=22, p<0.0001$; Fig. $3 A, B)$. Given that all treatments affected GLR-1 transport to a similar degree, we used $50 \mathrm{~nm}$ of $\mathrm{H}_{2} \mathrm{O}_{2}$ in all following experiments. In addition, quantification of GLR-1 transport in ctl-2(lf) mutants revealed a significant decrease in GLR-1 transport events $(12.3 \pm 1.10$ events per kymograph, $n=28, p=0.0012$, compared with $19.0 \pm 1.71$ events in controls, $n=20$; Fig. $3 C, D)$. The $\mathrm{H}_{2} \mathrm{O}_{2}$ treatment also led to significantly decreased anterograde transport velocities $(1.31 \pm 0.02 \mu \mathrm{m} / \mathrm{s}, \quad n=107$ events, $p<0.0001)$ compared with untreated controls (1.52 \pm 0.03 $\mu \mathrm{m} / \mathrm{s}, n=90$ events; Fig. $3 E, F)$. The ctl-2(lf) mutation caused a $17 \%$ decrease in anterograde transport velocity $(1.10 \pm 0.02 \mu \mathrm{m} /$ s, $n=107$ events, $p<0.0001)$ compared with controls $(1.33 \pm$ $0.02 \mu \mathrm{m} / \mathrm{s}, n=59$; Fig. $3 G, H)$. Neither $\mathrm{H}_{2} \mathrm{O}_{2}$ treatment nor $c t l-2$ (lf) altered retrograde transport velocity (data not shown), so remaining velocity analyses were focused on that of anterograde transport. In addition, the percent of time GLR-1-containing vesicles spent paused was decreased by these modest elevations in ROS levels $(p<0.0001$; Fig. 3I,J). Thus, both acute and chronic increase of $\mathrm{H}_{2} \mathrm{O}_{2}$ led to decreased transport of GLR-1containing vesicles to and from the cell body, as would be predicted by our observations of decreased somatic calcium in response to modest ROS elevations. Furthermore, these slight increases in ROS were sufficient enough to alter the normal characteristics of vesicle transport, including transport velocity as well as the frequency and/or duration of stops along the neuronal process.
Together, these changes in GLR-1 transport could impact GLR-1 delivery and exocytosis at synapses. To test this hypothesis, we turned to FRAP using GLR-1 tagged with mCherry and SEP (a pH-sensitive for of GFP) at the N-terminus (Fig. 4A) as previously described (Kennedy et al., 2010; Hoerndli et al., 2013). We monitored recovery of mCherry signal from the dual-tagged GLR-1 after bleaching to quantify new delivery of GLR-1 to synaptic sites in the proximal region of the AVA processes. SEP fluorescence, on the other hand, is quenched while in acidic endosomes and therefore protected from photobleaching (Kennedy et al., 2010; Hoerndli et al., 2015), meaning its signal is revealed once released to the membrane, providing a measure of exocytosis of GLR-1-containing receptors to synapses (Fig. $4 A$ ). Worms were again pretreated for 5 min with $50 \mathrm{nM} \mathrm{H}_{2} \mathrm{O}_{2}$ and then immediately mounted for imaging without any change in solution. During treatment, the rate of GLR-1 delivery was significantly decreased (as determined by the nonlinear fit of the percentage of fluorescence recovery throughout the 16 min following photobleaching, $n=10, p=0.0011$ ) compared with untreated controls $(n=10$; Fig. $4 B, C)$. Similar to the acute $\mathrm{H}_{2} \mathrm{O}_{2}$ treatment, ctl-2 (lf) also led to a significant reduction in the rate of synaptic GLR-1 delivery $(n=10, p<0.0001$; Fig. $4 B, C)$. This decrease in delivery could in part be because of the decreased time in which transported vesicles are stopped when ROS is elevated (Fig. 3I,J), which likely perturbs the ability to be delivered to synaptic sites. Together, these results show that even a modest, acute elevation of ROS can lead to decreased GLR-1 delivery at synapses.

The efficacy of excitatory neurotransmission is determined in part by the number of AMPARs at the surface of synapses (Huganir and Nicoll, 2013; Henley and Wilkinson, 2016); and although ROS elevation decreased GLR-1 delivery to synapses, it may not affect exocytosis rates or the number of receptors at the synaptic membrane. To determine whether this is the case, we quantified the SEP signal following GLR-1 photobleaching in both acute and genetic conditions of ROS elevation. Interestingly, the exocytosis rate of GLR-1 seemed to be unaffected by acute $\mathrm{H}_{2} \mathrm{O}_{2}$ treatment, whereas ctl-2(lf) significantly decreased GLR-1 exocytosis rates ( $n=10$ for all groups, $p<0.0001$; Fig. $4 D, E)$. This difference in SEP recovery suggests that acute and chronic ROS elevation differentially affect GLR-1 exocytosis at synapses. Chronic ROS elevation could lead to sustained decreases in GLR1 delivery, resulting in a time-dependent depletion of the synaptic reserves required for the GLR-1 exocytosis. If true, then a marked decrease in SEP::mCherry::GLR-1 signal at steady state in $c t l-2(l f)$ mutants would be expected. Surprisingly, measurements of SEP:: mCherry::GLR-1 fluorescence along the neuronal process before FRAP in $c t l-2(l f)$ and controls did not show a significant change in mCherry or SEP signal (data not shown). We postulate that the overexpression of GLR-1 necessary to follow single-vesicle dynamics might mask changes in steady-state levels of synaptic AMPARs.

To quantify the effect of physiological elevation of ROS levels in $c t l-2$ (lf) mutants on global glutamatergic circuit function and circumvent potential overexpression issues of the SEP::mCherry:: GLR-1 in AVA, we turned to behavioral analysis. The spontaneous reversal of $C$. elegans animals has been shown to reflect the function and number of synaptic GLR-1 receptors (Y. Zheng et al., 1999; Burbea et al., 2002; Park et al., 2009; Monteiro et al., 2012). In addition, AVA activation has been shown to be essential for spontaneous reversals (Gray et al., 2005; Ben Arous et al., 2010). Thus, we obtained reversal data for ctl-2(lf) and found that $c t l-2(l f)$ mutants exhibited fewer spontaneous reversals 
A

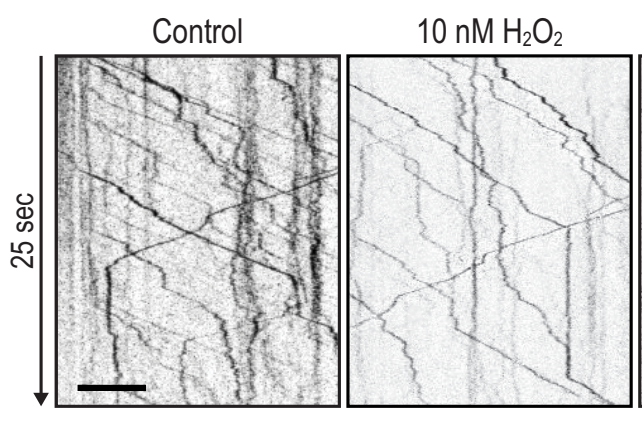

C

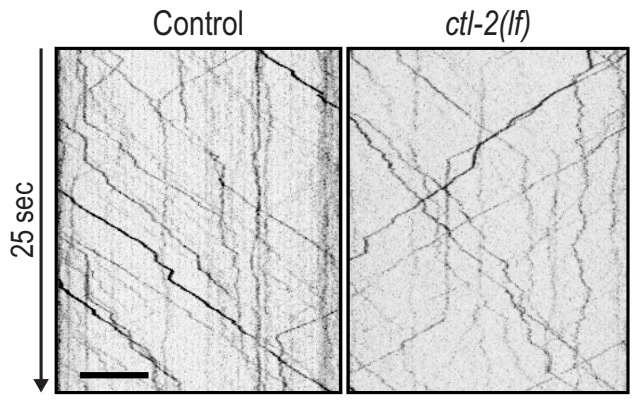

D

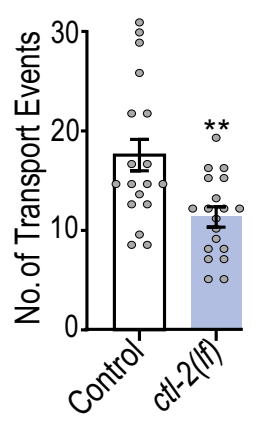

B

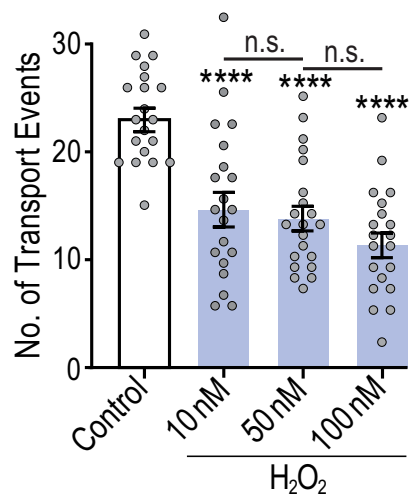

F
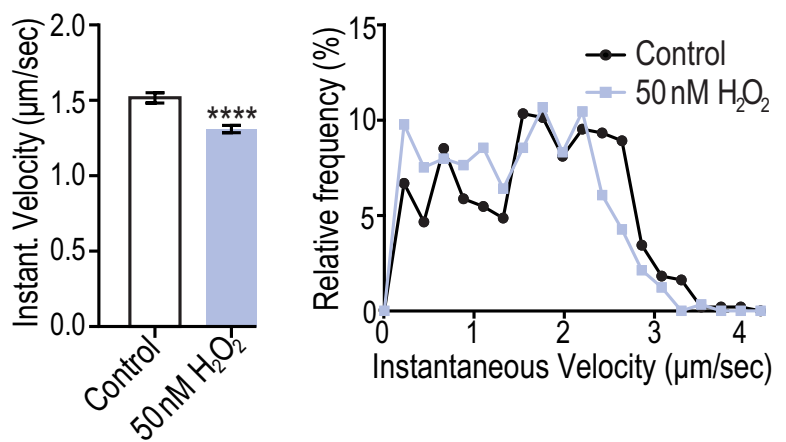

J
G

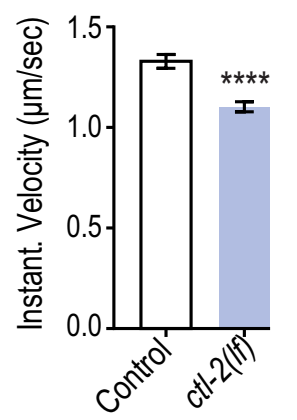

H

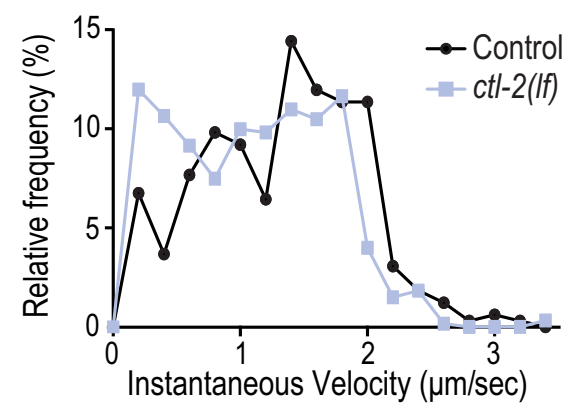

I

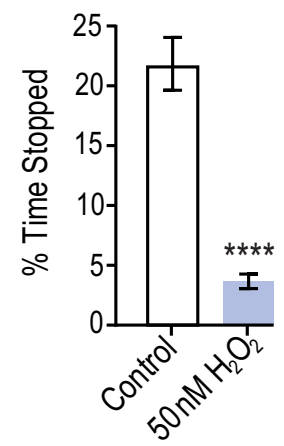

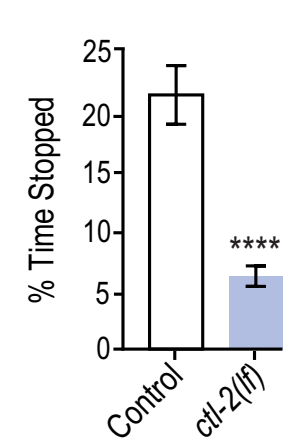

Figure 3. Physiologic increase in ROS levels cause decreased GLR-1 transport and alters transport dynamics. For all transport experiments, each group is expressing SEP:::mCherry::GLR-1 in the glr-1(ky176) background, and groups not subject to $\mathrm{H}_{2} \mathrm{O}_{2}$ treatment or additional mutations are referred to as "controls." $\mathrm{A}$, Representative kymographs from controls, 10,50 , and $100 \mathrm{~nm}$ $\mathrm{H}_{2} \mathrm{O}_{2}$-treated worms. Scale bar, $5 \mu \mathrm{m}$. B, Quantification of total transport events from $50 \mathrm{~s}$ recordings from control $(n=22), 10(n=20), 50(n=21)$, and $100 \mathrm{~nm} \mathrm{H}_{2} \mathrm{O}_{2}$-treated $(n=22)$ worms. n.s. $=$ not significant, ${ }^{* * * *} p<0.0001$. C, Representative kymographs from controls and $c t l-2(I f)$ mutants. $\boldsymbol{D}$, Quantification of total transport events in controls $(n=21)$ and $c t l-2(I f)(n=28)$. ${ }^{* *} p=0.0012$. $E$, G, Instantaneous velocity analysis of anterograde transport events in $(\boldsymbol{E})$ control and $50 \mathrm{~nm} \mathrm{H}_{2} \mathrm{O}_{2}$-treated worms ( $n>90$ events; ${ }^{* * * *} p<0.0001$ compared with controls) or (G) controls and $c t l-2(I f)$ mutants $\left(n>50\right.$ events; ${ }^{* * * *} p<0.0001$ compared with controls). $\boldsymbol{F}, \boldsymbol{H}$, Frequency distribution of the anterograde instantaneous velocities (binned every $0.2 \mu \mathrm{m} / \mathrm{s}$ ) in $(\boldsymbol{F})$ controls and $50 \mathrm{~nm} \mathrm{H}_{2} \mathrm{O}_{2}$-treated worms or $(\boldsymbol{H})$ controls and ctl-2(If) mutants. I, J, Percent of time GLR-1 vesicles spent stopped in each group. ${ }^{* * * *} p<0.0001$, compared with controls.

$(1.37 \pm 0.19$ reversals per minute, $p<0.0001, n=38)$ compared with controls ( $3.78 \pm 0.26, n=37$, data not shown). This behavioral change supports our calcium imaging data in which the total spontaneous activity of AVA is decreased in ctl-2(lf). Together, our results clearly show that modest ROS elevation is sufficient to modify synaptic GLR-1 transport, delivery, and, with chronic elevations, exocytosis to synapses ultimately affecting glutamatergic circuit function.

\section{Increased ROS modulate GLR-1 transport at or directly downstream of L-type VGCC}

Our results indicate that modest increases in ROS (within the physiological range for neurons) (Sies, 2017) decrease the activity-dependent fluctuations in cytoplasmic calcium in the AVA interneurons of C. elegans (Fig. 2). This results in decreased GLR-1 transport into and out of the cell body as well as decreased anterograde transport velocity and time vesicles spent stopped along the neurite (Fig. 3). Together, these changes in transport likely contribute to decreased delivery and, in the case of prolonged elevations in ROS, exocytosis of GLR-1 at synapses (Fig. 4). To add clarity, we investigated mechanisms by which excess ROS decreases cytoplasmic calcium, CaMKII activation, and therefore GLR-1 transport.

Our data presented above suggest a regulatory signaling pathway (Fig. $5 A$ ), in which depolarization of AVA via glutamate receptor activation leads to calcium influx through VGCCs to activate CaMKII. This model led us hypothesize that elevated 
A

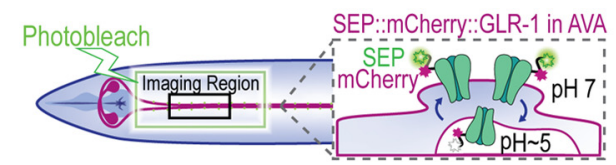

B

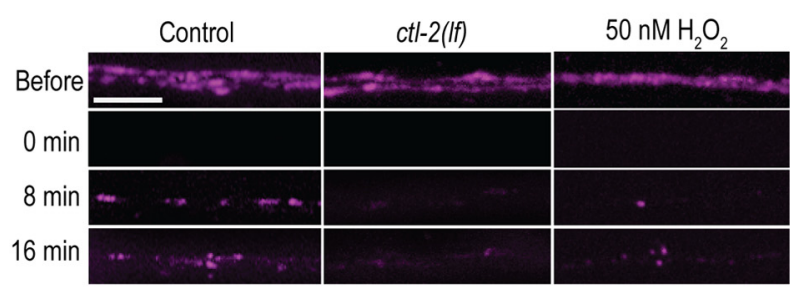

D

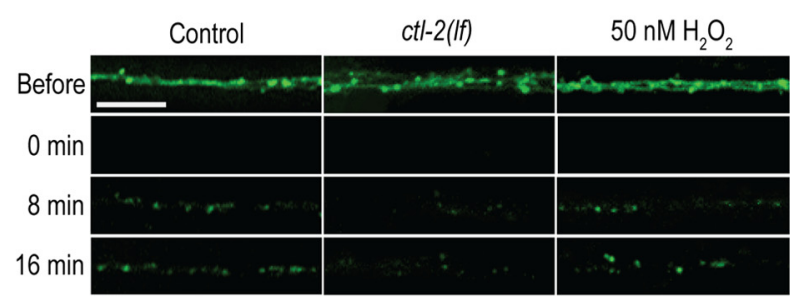

C

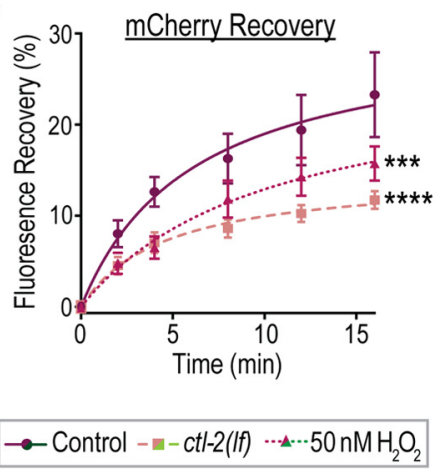

E

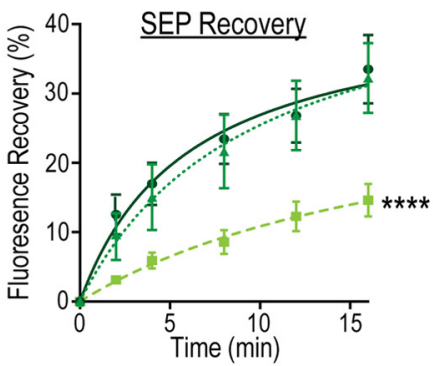

Figure 4. Physiologic increase in ROS cause decreased GLR-1 delivery and exocytosis to synapses. $\boldsymbol{A}$, Illustration of imaging location of FRAP in the AVA interneurons expressing GLR-1 tagged with SEP, a pH-sensitive GFP, and mCherry. All experimental groups express this dual-tagged GLR-1 in the AVA in the glr-1(ky176) background. Groups that were not subject to $\mathrm{H}_{2} \mathrm{O}_{2}$ treatment or additional mutations are referred to as "control." $\boldsymbol{B}$, Representative maximum projections of the mCherry fluorescence in the AVA interneurons before, immediately after ( 0 min), 8 and $16 \mathrm{~min}$ after photobleaching of the imaging region. Scale bar, $5 \mu \mathrm{m}$. $\mathrm{C}$, Percent recovery of mCherry fluorescence after photobleaching over time in control, untreated $\mathrm{ct} /-2(\mathrm{If})$, and $50 \mathrm{nM} \mathrm{H}_{2} \mathrm{O}_{2}$ treated worms ( $n=10$ for each group). ${ }^{* * *} p=0.001,{ }^{* * *} p<0.0001$, compared with controls. $\boldsymbol{D}$, Representative maximum projections of the SEP fluorescence in the AVA interneurons before, immediately after, 8 and 16 min after photobleaching of the imaging region. Scale bar, $5 \mu \mathrm{m}$. $\boldsymbol{E}$, Percent recovery of SEP fluorescence compared with before photobleaching from the same worms as in Figure 4C. ${ }^{* * *} p<0.0001$, compared with controls.

ROS could be affecting calcium signaling needed for CaMKII activation. We used a genetic epistasis strategy to test whether this is a linear pathway in which ROS function upstream of CaMKII activation to modulate GLR-1 transport. We made double mutants with $u n c-43(l f)$ and $c t l-2(l f)$ as well as $u n c-43(g f)$ and ctl-2(lf). If ROS are acting upstream of CaMKII activation, then one would predict that $u n c-43(g f / l f)$; ctl-2(lf) double mutants would be similar to the single unc-43 mutations alone.

To test this, we analyzed mCherry::GLR-1 transport and again observed that $c t l-2(l f)$ alone decreases GLR-1 transport, but interestingly, the addition of the $c t l-2(l f)$ to $u n c-43$ (lf or $g f$ ) mutations does not change the amount of transport compared with unc-43 mutations alone (Fig. 5B,C). Analysis of anterograde transport velocity and stopping in the unc-43(gf) mutants mirrors these results in that the velocity and stopping are the same in the unc43(gf); ctl-2(lf) as in the $u n c-43(g f)$ single mutant (Fig. $5 D, E)$. It is interesting to note that the addition of the $c t l-2(l f)$ mutation to $u n c-43(g f)$ did not affect transport velocity compared with unc43( $g$ ), which indicates that modest ROS elevation likely does not lead to global perturbations of cellular processes, which would indirectly affect molecular motor-dependent activity. To determine whether this is unique to $c t l-2(l f)$, we also treated $u n c-43(g f)$ animals with $50 \mathrm{~nm} \mathrm{H}_{2} \mathrm{O}_{2}$ before imaging transport. Similar to ctl-2(lf), acute treatment with $\mathrm{H}_{2} \mathrm{O}_{2}$ had no effect on the unc-43 (gf)-mediated increase in GLR-1 transport (Fig. 5F,G). Together, these data indicate that ROS signaling regulates GLR-1 transport upstream of CaMKII activation.

To pinpoint potential targets of ROS upstream of CaMKII activation, we next investigated L-VGCCs, which we have shown to play an important role in regulating GLR-1 transport events and delivery (Fig. 1). To determine whether L-VGCCs or downstream calcium signaling is a target of ROS modulation, we used a genetic epistasis approach. We made double mutants with $\mathrm{ctl}-2$ (lf) and either egl-19(rf) or egl-19(gf) and then analyzed GLR-1 transport. These analyses reveal that the amount of GLR-1 transport in egl-19(rf); ctl-2(lf) is not significantly different from egl-19 ( $r f$ ) (Fig. 6A,B). However, egl-19(gf); ctl-2(lf) mutants have significantly decreased GLR-1 transport events compared with egl-19 (gf) alone $(13.9 \pm 1.17$ vs $26.7 \pm 1.76$, respectively; $n=16$, $p<0.0001$; Fig. $6 A, B)$. Indeed, the double mutant was not significantly different from $c t l-2(l f)$ alone (11.2 \pm 0.91 events, $n=19$, $p=0.42$; Fig. $6 A, B)$. Quantification of the anterograde velocity and stopping of transport events in these strains further support the idea that ROS affect calcium signaling at or just downstream of L-VGCCs. In the egl-19(gf) mutant, instantaneous velocity of anterograde transport is increased compared with controls $(1.37 \pm 0.03$ vs $1.23 \pm 0.02 \mu \mathrm{m} / \mathrm{s}, n>40$ events, $p=0.0005$; Fig. $6 C, D)$. When combined with $c t l-2(l f)$, anterograde transport velocity is decreased to around that of the ctl-2(lf) mutation alone $(1.12 \pm 0.03, n=43$, vs $1.04 \pm 0.02, n=82, p=0.66$; Fig. $6 C, D)$. The percent of time GLR-1 vesicles spent stopped along the neuronal process was increased in the egl-19(gf) mutants (stopped $29.9 \pm 2.72 \%$ of time, $n=96)$ compared with controls $(20.8 \pm$ $1.86 \%, n=95, p=0.044$; Fig. $6 F)$. When combined with $c t l-2(l f)$, stopping was drastically decreased compared with egl-19(gf) alone $(11.65 \pm 1.94 \%, n=44, p<0.0001)$ and controls $(20.88 \pm$ $1.86 \%, n=89, p=0.015$; Fig. $6 F$ ). These data suggest that elevated ROS change transport velocity and stopping throughout the neurite via decreased calcium. If true, then we hypothesized that transport velocity and stopping should be decreased in the egl-19 (rf) mutant. 
A Proposed Calcium Signaling Pathway Regulating AMPAR Transport:

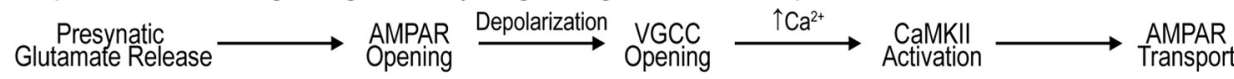

B

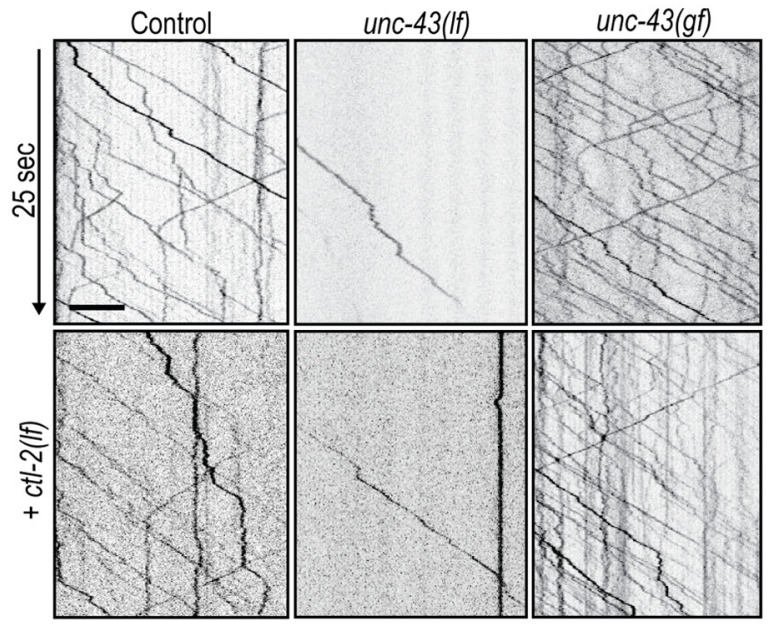

C

D

E
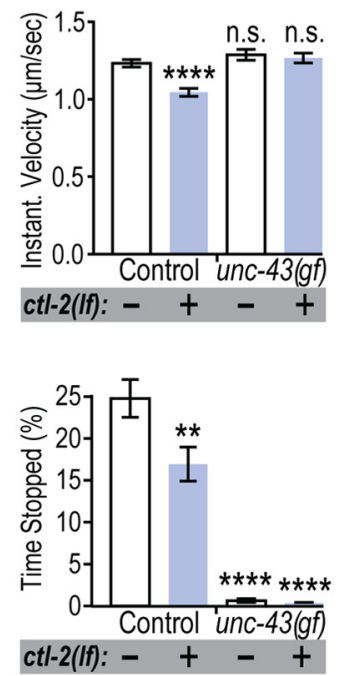

$\mathbf{F}$
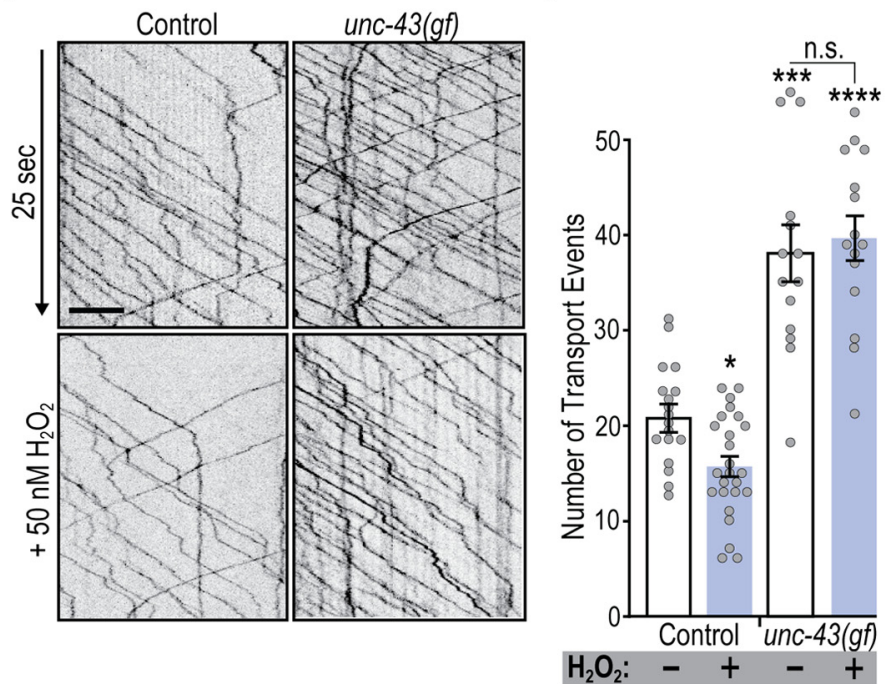

Figure 5. Increased ROS acts on GLR-1 transport upstream of CaMKII activation. $\boldsymbol{A}$, Previously proposed model of an activity-dependent calcium signaling pathway regulating AMPAR transport (Hoerndli et al., 2015). All experimental groups express SEP::mCherry::GLR-1 in the glr-1(ky176) background. B, 25 s of representative kymographs from each experimental group. Scale bar, $5 \mu \mathrm{m}$. C, Quantification of transport events from full-length kymographs (50 s) in control, unc-43(If), and unc-43(gf) without (-, white bars) and with (+, blue bars) the ctt-2(If) mutation $(n \geq 13)$. n.s. $=$ not significant, ${ }^{* *} p=0.0012,{ }^{* * * *} p<0.0001$, compared with controls lacking the ctl-2(If) mutation. $\boldsymbol{D}$, Instantaneous velocity of anterograde transport for the same animals from $\boldsymbol{B}$ and $\boldsymbol{C}\left(n>60\right.$ events). n.s. $=$ not significant, ${ }^{* * * *} p<0.0001$, compared with controls lacking the ctl-2(If) mutation. $\boldsymbol{E}$, Percent of time GLR-1 vesicles spent stopped in these same genotypes $\left(n>60\right.$ events). ${ }^{* *} p=0.009$, ${ }^{* * *} p<0.0001$, compared with controls lacking the ctl-2(If) mutation. $\boldsymbol{F}, 25 \mathrm{~s}$ of representative kymographs from each experimental group. Scale bar, $5 \mu \mathrm{m}$. G, Quantification of transport events in control or unc $-43(\mathrm{gf})$ with (+, blue bars) or without $\left(-\right.$, white bars) $50 \mathrm{~nm} \mathrm{H}_{2} \mathrm{O}_{2}$ treatment $(n \geq 14)$. n.s. $=$ not significant, ${ }^{*} p=0.025$, ${ }^{* * *} p=0.0002,{ }^{* * *} p<0.0001$, compared with untreated control.

Similar to our quantification of GLR-1 transport numbers, the transport velocity of $e g l-19(r f)$ is decreased compared with controls $(1.08 \pm 0.04 \mu \mathrm{m} / \mathrm{s}, n=86, p=0.0001)$ and is unaffected by the addition of the $c t l-2(l f)$ mutation (Fig. $6 C, E$ ). The percentage of time vesicles were stopped was also decreased in egl-19(rf) mutants (14.93 $\pm 2.15 \%, n=79, p=0.001$; Fig. $6 F)$. However, in contrast to our velocity results, we observed a slight, but not significant, decrease in stopping with the addition of ctl-2(lf) (8.7 \pm $1.60 \%, n=67, p=0.43$; Fig. $6 F$ ). These data indicate that the $c t l-2$ ( $\mathrm{lf}$ ) mutation is acting in the same pathway as egl-19 to regulate GLR-1 transport. To ensure that these results are because of elevated ROS levels and not developmental effects of $c t l-2(l f)$, we also treated $e g l-19(g f)$ animals with $50 \mathrm{~nm}_{2} \mathrm{O}_{2}$. As previously observed, $\mathrm{H}_{2} \mathrm{O}_{2}$ treatment of control animals reduced the amount of GLR-1 transport and reduced egl-19(gf) transport to the same level as $\mathrm{H}_{2} \mathrm{O}_{2}$ alone $(26.11 \pm 1.15$ vs $18.9 \pm 1.76$ events, $n>18$; Fig. $6 G, H$ ). To eliminate the possibility of these results being caused by developmental changes in the egl-19(gf/rf) mutants, we also analyzed GLR-1 transport in control and ctl-2 (lf) mutants following treatment with the L-VGCC blocker, nemadipine. As we previously showed, a $10 \mu \mathrm{m}$ nemadipine treatment significantly reduced the total amount of GLR-1 transport (14.36 \pm 1.53 events, $n=11$, compared with controls $24.00 \pm 2.15$ events, $n=10 ; p=0.008$; Fig. $6 I, J)$. However, nemadipine treatment did not further reduce transport in $c t l-2(l f)$ mutants $(14.56 \pm 1.13$ events, $n=9$, in 
A

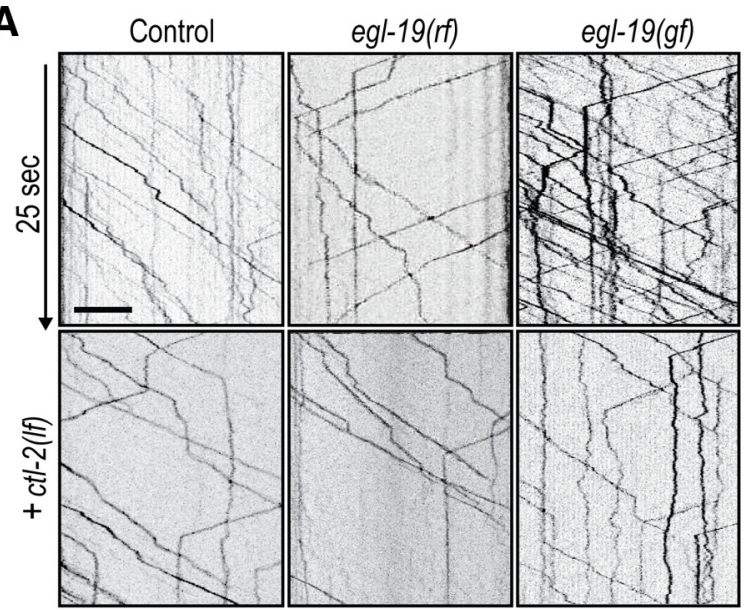

B

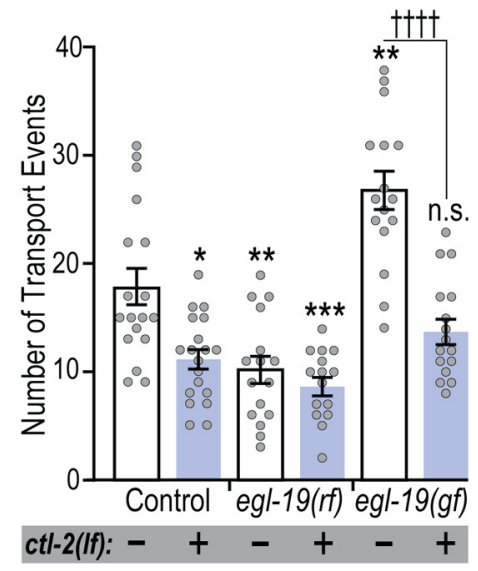

C

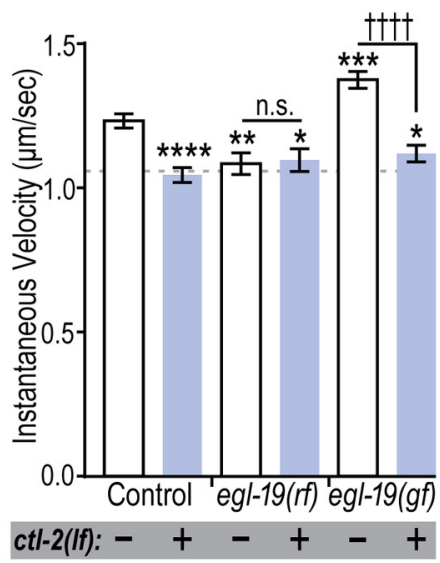

D

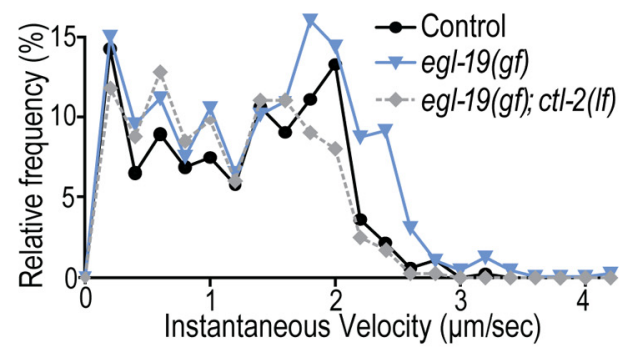

G

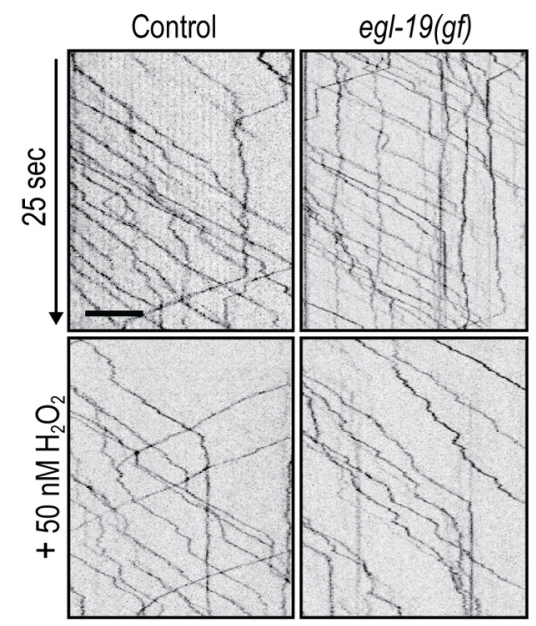

H
E

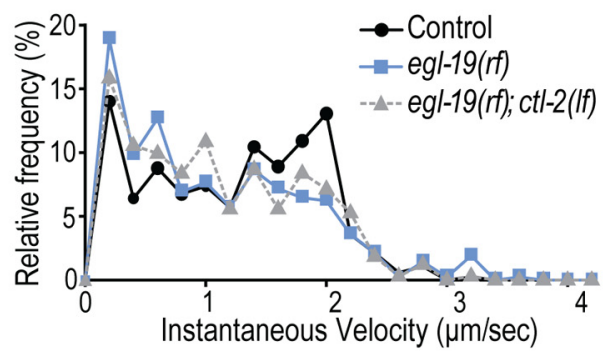

F

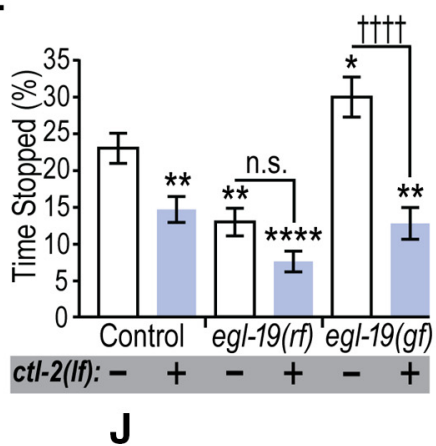

I
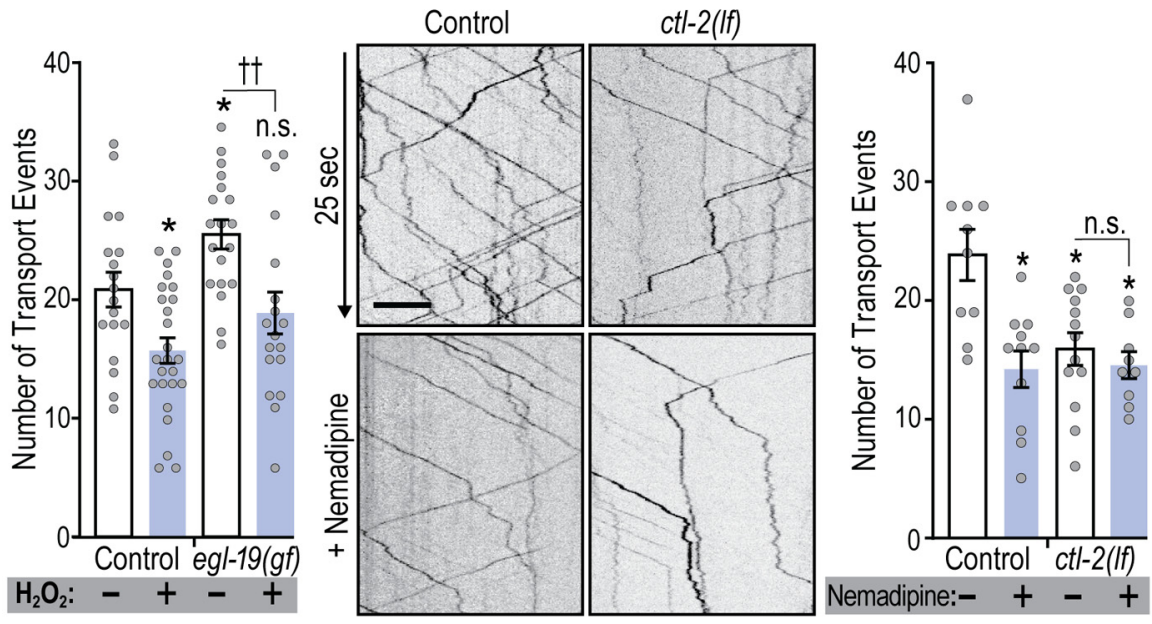

Figure 6. ROS decrease GLR-1 transport numbers and alter transport dynamics by acting on or downstream of L-VGCCs. All experimental groups express SEP::mCherry::GLR-1 in the glr-1 (ky176) background. $\boldsymbol{A}, \mathbf{G}, \boldsymbol{I}, 25 \mathrm{~s}$ from representative kymographs from each experimental group. Scale bar, $5 \mu \mathrm{m}$. B, Quantification of transport events from controls, egl-19(rf), or egl-19(gf) without $\left(-\right.$, white bars) or with $\left(+\right.$, blue bars) the ctl-2(If) mutation $(n \geq 15)$. n.s. $=$ not significant, ${ }^{*} p=0.012,{ }^{* *} p=0.007$, ${ }^{* * *} p=0.0005$, compared with controls lacking the ctl-2(If) mutation. ${ }^{\mathrm{t} t+1} p<0.0001$, compared with egl-19(gf) alone. C, Instantaneous velocity of anterograde transport events in each group $\left(n \geq 44\right.$ events). n.s. $=$ not significant, ${ }^{*} p<0.05$, ${ }^{* *} p=0.0035,{ }^{* * *} p=0.0005,{ }^{* * * *} p<0.0001$, compared with controls lacking the ctl-2(If) mutation. ${ }^{+t t t} p<0.0001$, compared with egl-19(gf) alone. D, E, Frequency distribution of instantaneous velocity (binned every $0.2 \mu \mathrm{m} / \mathrm{s}$ ) of anterograde transport events for egl-19(gf) single and double mutants $(\boldsymbol{D})$ as well as egl-19(rf) single and double mutants $(\boldsymbol{E})$ compared with controls lacking $c t \mid-2(I f)$. $\boldsymbol{F}$, Percent of time each GLR-1 vesicle spent stopped for each group $\left(n \geq 44\right.$ events). n.s. $=$ not significant, ${ }^{*} p=0.044,{ }^{* *} p \leq 0.009,{ }^{* * * *} p<0.0001$, compared with controls lacking $\mathrm{ct}-2$ (If). ${ }^{\mathrm{tttt} p}<0.0001$, compared with egl-19(gf) alone. $\boldsymbol{H}$, Quantification of transport events from controls and egl-19(gf) without $\left(-\right.$, white bars) or with $\left(+\right.$, blue bars) $50 \mathrm{~nm} \mathrm{H}_{2} \mathrm{O}_{2}$ treatment $(n \geq 18)$. n.s. $=$ not significant, ${ }^{*} p \leq 0.038$, compared with untreated controls. ${ }^{+\dagger} p=0.007$, compared with untreated egl-19(gf). J, Quantification of transport events in controls and $c t-2(I f)$ with $\left(+\right.$, blue bars) and without $\left(-\right.$, white bars) $10 \mu$ m nemadipine treatment $(n>10)$. n.s. $=$ not significant, ${ }^{*} p<0.05$, compared with untreated controls.

nemadipine-treated animals compared with $15.92 \pm 1.38$ events, $n=13$, in untreated controls; Fig. $6 I, J)$. Together, these observations suggest that ROS elevation is affecting LVGCC/EGL-19-dependent calcium signaling.

To obtain additional insight into how ROS modulate GLR-1 transport and delivery, we combined FRAP of SEP::mCherry:: GLR-1 and our genetic epistasis strategy (Fig. 7). We quantified synaptic delivery of GLR-1 using FRAP of mCherry signal (Fig. $7 A, B$ ) and synaptic exocytosis using FRAP of SEP signal (Fig. 7C, $D)$ in single egl-19(rf) or egl-19(gf) mutants and when doubled with $c t l-2(l f)$. Similar to our GLR-1 transport results, quantification of mCherry and SEP FRAP showed that the rate of GLR-1 delivery and exocytosis is decreased in egl-19(rf) mutants and addition of $c t l-2(l f)$ causes no change in those rates $(n=10$; 
A

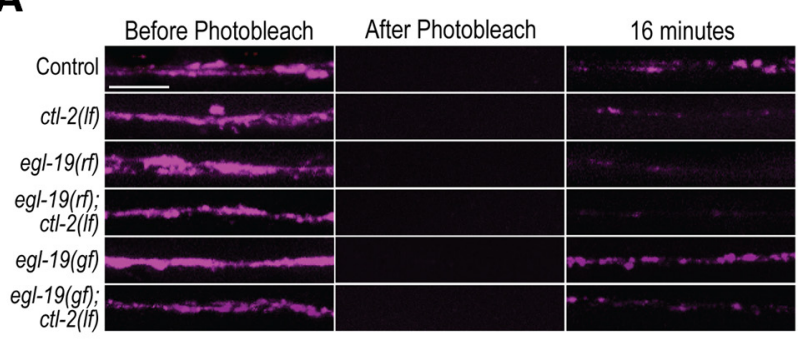

C

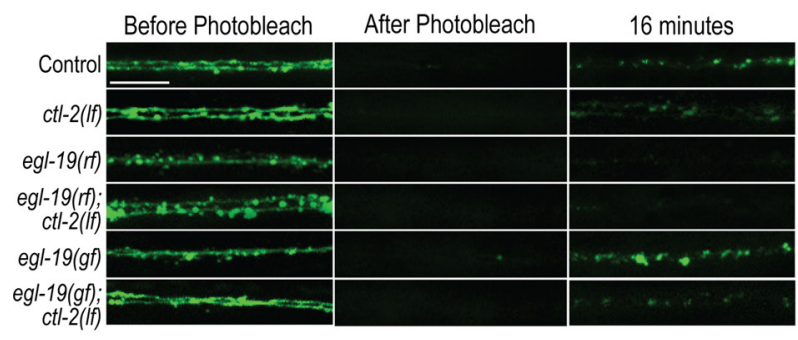

B

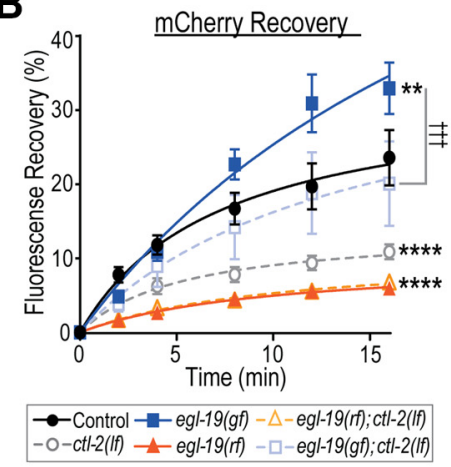

$\mathbf{D}$

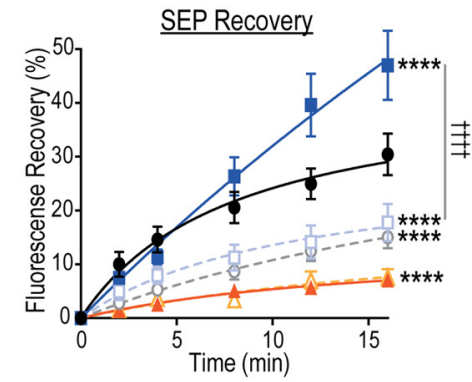

Figure 7. ROS decrease GLR-1 synaptic delivery and exocytosis by acting on or downstream of L-VGCCs. All experimental groups express mCherry::SEP::: GLR-1 in the AVA in the glr-1(ky176) background. Groups without additional mutations are referred to as "control." $A$, Representative maximum projections of the mCherry fluorescence in the AVA interneurons before, immediately after, and $16 \mathrm{~min}$ after photobleaching of the imaging region from each experimental group. Scale bar, $5 \mu \mathrm{m}$. $\boldsymbol{B}$, Recovery of mCherry fluorescence following photobleaching in control, egl- 19

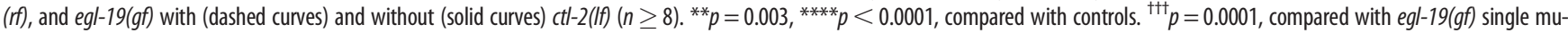
tant. $\boldsymbol{C}$, Representative maximum projections of the SEP fluorescence in the same groups. Scale bar, $5 \mu \mathrm{m}$. $\boldsymbol{D}$, SEP fluorescence recovery in the same experimental groups. ${ }^{* * *} p<0.0001$, compared with controls. ${ }^{\mathrm{ttt} p} p<0.0001$, compared with egl-19(gf).

Fig. 7). Alternatively, the FRAP of mCherry in egl-19(gf); ctl-2(lf) is reduced compared with egl-19(gf), but not identical to $c t l-2(l f)$ (Fig. $7 B$ ) as is the case with the FRAP of SEP in these genotypes (Fig. 7D). These results suggest that ROS not only modulate GLR-1 transport, but also delivery and exocytosis by affecting the signaling cascade initiated by calcium influx through LVGCC/EGL-19.

Our data provide a better understanding of the mechanism by which activity-dependent calcium signaling regulates AMPAR transport to ultimately affect synaptic delivery and exocytosis of AMPARs. We go on to show that L-VGCCs play a central role in regulating cytoplasmic calcium dynamics and that this process is modified by ROS. This leads to our suggested model (Fig. 8) in which physiological elevations of ROS can modulate synaptic GLR-1 transport at the soma and in the dendritic process by modifying calcium signaling originating to a large extent from L-VGCCs.

\section{Discussion}

Activity-dependent calcium signaling is required for AMPAR transport in vertebrate and C. elegans glutamatergic neurons (Hoerndli et al., 2015; Hangen et al., 2018). Here we show that in C. elegans, calcium influx mediated by L-VGCC/EGL-19 is not only required, but that the magnitude of calcium influx directly corresponds to changes in AMPAR transport to and from the cell body as well as AMPAR transport dynamics within neuronal processes. Furthermore, we show that physiological increases in ROS decrease somatic calcium signaling (Fig. 2) causing a subsequent decrease in somatic AMPAR export and synaptic delivery (Fig. 3). This study reveals a physiological interplay between ROS and calcium signaling that directly affects AMPAR transport and delivery (Fig. 8). It also prompts fundamental questions regarding how AMPAR transport is regulated by activity in a spatially specific manner, such as how do somatic versus local dendritic calcium signaling pathways regulate AMPAR transport and what are their effects on synaptic function and plasticity?

Long-distance AMPAR transport is one of the least characterized components of the multistep trafficking of AMPAR from the cell body to the periphery (Henley and Wilkinson, 2016). It is clear that transport requires molecular motors, including kinesin-1 (C. H. Kim and Lisman, 2001; Setou et al., 2002; Hoerndli et al., 2013), and is regulated by neuronal activity and CaMKII (Esteves da Silva et al., 2015; Hoerndli et al., 2015; Hangen et al., 2018). However, we do not understand the roles of other key players required for activity-dependent regulation and at which steps (i.e., cargo loading, transport, delivery, and removal) they act. Consistent with previous reports (Hoerndli et al., 2015), we show decreased somatic export of AMPARs in mutants with reduced L-VGCC function or after treatment with the L-VGCC blocker, nemadipine (Figs. 1, 6). On the contrary, increased calcium influx in gain-of-function L-VGCC mutants leads to more AMPAR transport demonstrating that activity-dependent calcium influx through L-VGCCs bidirectionally regulates AMPAR transport out of the cell body (Fig. 1).

Once motors and cargoes have entered the dendrites, they are subject to the conditions of those compartments. For instance, GLR-1 transport in the AVA neurite is bidirectional and heterogeneous, displaying increased stopping probabilities at synaptic localizations of GLR-1 (Hoerndli et al., 2013). Several studies suggest that stopping of transport vesicles correlate with delivery of AMPARs to synapses (Hoerndli et al., 2013; Hangen et al., 2018), but it is unclear how that is regulated and if vesicular stops 


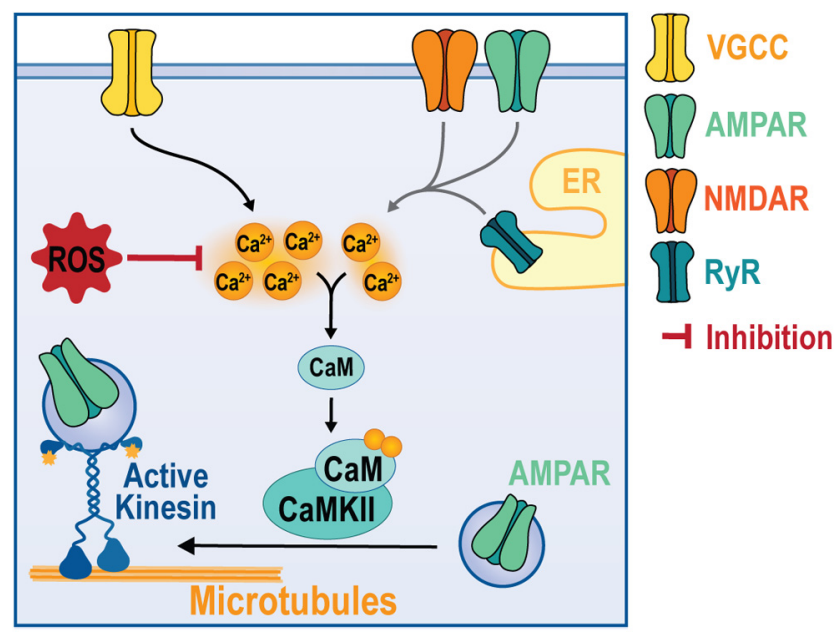

Figure 8. Proposed ROS signaling effect on GLR-1 transport. Model showing that increases in ROS inhibit the activity-dependent changes in cytoplasmic calcium, which occur mostly because of calcium influx through L-VGCC (yellow channel, VGCC), but also AMPARs (green channel) and NMDARs (orange channel). Intracellular release of calcium, such as from the ER via ryanodine receptors (blue channel, RyR), can also contribute to cytoplasmic elevations in calcium. As a consequence, decreased cytoplasmic calcium limits calmodulin (CaM) binding and activation of CaMKII, reducing the number of AMPAR transport and delivery to synapses.

affect synaptic delivery. Here, we demonstrate that manipulations of calcium influx through L-VGCC modify AMPAR transport dynamics, such as stops and velocity profiles (Fig. 1), and lead to changes in synaptic delivery (Fig. 7). We show that decreased calcium influx through L-VGCCs decreases anterograde velocity and stopping of AMPAR-containing vesicles within the neurite. Conversely, increasing calcium influx through L-VGCC led to increased anterograde velocities and stops (Fig. 1 ). Decreased velocity and stopping corresponds to less GLR-1 delivery and insertion to synapses, whereas higher velocities and stops correspond to higher delivery and insertion (Figs. 1, 7), which is consistent with previous findings (Hoerndli et al., 2013; Hangen et al., 2018) and demonstrates that regulation of AMPAR transport dynamics in dendrites is a key factor in controlling AMPAR delivery and synaptic function. Interestingly, there is a drastic decrease in stop frequency in unc-43(gf) mutants in which CaMKII is no longer regulated by calcium, suggesting that stop frequency of GLR-1 transport could be mediated by calcium-dependent changes in CaMKII activity. Together, these results provide evidence for a model in which calcium signaling controlled by L-VGCCs and CaMKII regulate AMPAR transport into and out of the cell body as well as in the dendritic process in an activity-dependent manner.

The AVA interneurons have long, relatively flat processes in the ventral cord that enable high-resolution, time-lapse imaging and cell-specific expression using the AVA-specific promoters rig-3 and flp-18. Although powerful, this analysis is limited to AVA interneurons, which leaves open the possibility that other glutamatergic neurons, such as those with specific compartmentalized calcium signaling (Hendricks et al., 2012), might have different ROS sensitivity thresholds or regulatory mechanisms. However, our results using this approach concur with studies using vertebrate neurons in which long-term increases in dendritic calcium increased the amount of AMPAR transport and delivery (Hangen et al., 2018). Nevertheless, our data are a new, in vivo demonstration of activity-dependent calcium signaling bidirectionally regulating AMPAR transport throughout the neuron, determining transport vesicle numbers, dynamics, and delivery at synapses at short and long timescales.

Importantly, gain- or loss-of-function CaMKII mutants have a much stronger effect on GLR-1 somatic export than L-VGCC mutations alone. This could be because of the mutations in L-VGCCs not being a complete loss- or gainof-function. However, it could also suggest that additional signaling pathways converge onto CaMKII to regulate GLR-1 somatic export. These signaling pathways could involve other calcium sources (i.e., NMDAR and ryanodine receptors) or other signaling cascades, such as those involving protein kinase $\mathrm{A} / \mathrm{C}$ or mitogen-activated protein kinase (Boehm et al., 2006; Z. Zheng and Keifer, 2008; Ren et al., 2013; Eales et al., 2014; Tang and Yasuda, 2017). Although these signaling pathways change synaptic AMPAR trafficking, their roles in somatic export and/or long-distance transport dynamics are yet unknown.

\section{Compartmentalized calcium signaling}

Calcium signaling originating from the plasma membrane, ER, Golgi, or mitochondria at neuronal cell bodies, dendrites, and axons differentially regulate a variety of neuronal functions (for review, see Brini et al., 2014). Calcium influx in the dendritic shaft and dendritic spines comes from various ion channels, including AMPAR, NMDAR, and voltage-sensitive calcium channels localized to the membrane, ER, and mitochondria (Catterall, 2011; Higley and Sabatini, 2012). Calcium imaging in cell culture has shown calcium to remain within the spine following single spine activation (Sabatini et al., 2001; Nimchinsky et al., 2002). Interestingly, vertebrate and invertebrate neurons have compartmentalized calcium transients that are important for neuronal function and computation (Higley and Sabatini, 2012; Donato et al., 2019; Ali and Kwan, 2020). However, it is unclear how compartmentalization of calcium regulates downstream processes such as trafficking of synaptic proteins.

Compartmentalized calcium signaling is conserved in C. elegans interneurons (Hendricks et al., 2012; Donato et al., 2019). Our data show that AMPAR transport in the long process of AVA is affected by altered calcium influx because of mutations in L-VGCC and ROS levels. Some of these effects are likely to be local in scope. However, we have no knowledge regarding distribution of VGCCs, including EGL-19, or about compartmentalization of calcium signaling in the AVA. It is unclear how synaptic inputs and calcium signaling are integrated at dendritic and cellular levels to tailor AMPAR distribution. Our study provides a platform to start understanding in vivo calcium and ROS signaling mechanisms regulating AMPAR distribution, ultimately affecting synaptic strength and behavior of animals.

\section{ROS}

Neuronal excitation and synaptic plasticity have high energy demands and correlate with physiological fluctuations in ROS levels (Bindokas et al., 1996). Pathophysiological ROS elevation is observed in aging and neurodegenerative conditions, such as Parkinson's, Huntington's and Alzheimer's disease (Stefanatos and Sanz, 2018). Intriguingly, some amount of ROS, in particular superoxide $\left(\mathrm{O}_{2}{ }^{-}\right)$, is required for LTP induction and memory formation (Thiels et al., 2000). This suggests that ROS are required for LTP formation, supporting a necessary signaling role in excitatory neuronal function, perhaps specifically in AMPAR trafficking. However, there is currently no direct mechanistic link between ROS signaling and regulation of AMPAR trafficking. Our results show that physiological ROS elevation decreases 
AMPAR transport, delivery, and exocytosis (Fig. 3) through a mechanism involving decreased calcium influx (Fig. 2). Consistent with our results, hypoxic conditions triggering increases in ROS production lead to decreased GLR-1 trafficking in C. elegans (Park and Rongo, 2016).

Although there have been quite a few studies of the regulation of L-VGCC function by physiological ROS in several cell types (Chaplin and Amberg, 2012; Todorovic and Jevtovic-Todorovic, 2014; Cserne Szappanos et al., 2017), there have been extremely few in neurons (Massaad and Klann, 2011; Hidalgo and AriasCavieres, 2016; Wilson et al., 2018) and none in vivo. Our study is the first to show how small increases in ROS affect whole-cell activity-dependent calcium signaling and direct downstream targets, such as synaptic AMPAR transport and delivery. We show that genetically and pharmacologically induced increases in ROS within the range of physiological signaling levels $(\sim 50 \mathrm{nM})$ (Sies, 2017) lead to decreased calcium signaling in the cell bodies of $C$. elegans AVA neurons (Fig. 2). This contrasts with what has been found in vertebrate arterial smooth muscle and gonadotropes (Chaplin and Amberg, 2012; Dang et al., 2018), but is consistent with reports of mitochondrial ROS elevation inhibiting L-VGCCs in cardiomyocytes (Scragg et al., 2008). Discrepancies in the concentration of ROS treatment between these studies and the one presented here could explain the opposing results. Several cellular processes have been shown to be impacted differently by ROS levels within versus outside of the physiological range (Wilson and González-Billault, 2015; Beckhauser et al., 2016). It is possible that ROS regulation of calcium sources follows suit in that moderate increases in ROS cause decreased calcium influx, but greater increases in ROS cause increased calcium influx.

Together, our results identify a novel role for ROS signaling in the regulation of AMPAR transport and synaptic delivery, providing a link between the metabolic demands of neuronal activity and excitatory neurotransmission. Future studies are required to test our proposed model and understand its biological significance. Importantly, follow-up studies will first be aimed at determining the source and targets of activity-induced ROS signaling. The ability to answer these questions will rely on the development of high-affinity, genetically encoded ROS sensors enabling in vivo subcellular imaging of these dynamics. It is interesting to note that dysregulation of calcium signaling and ROS homeostasis is associated with aging and neurodegeneration (Barja, 2013; K. W. Kim and Jin, 2015; Grimm and Eckert, 2017; Stefanatos and Sanz, 2018). In this context, several C. elegans models of Alzheimer's disease have provided key in vivo evidence to demonstrate the causal role of calcium dysregulation in neuronal (Griffin et al., 2019) and muscular degeneration (Sarasija and Norman, 2015; Sarasija et al., 2018). Furthermore, it has been shown that neurodegenerative models in C. elegans are translationally relevant for vertebrate studies (Treusch et al., 2011). Thus, once a fundamental understanding of how ROS signaling normally regulates excitatory neuronal function has been obtained in our model, future studies will determine how ROS and excitatory neurotransmission change in aging and neurodegeneration.

\section{References}

Akaishi T, Nakazawa K, Sato K, Saito H, Ohno Y, Ito Y (2004) Modulation of voltage-gated $\mathrm{Ca}^{2+}$ current by 4-hydroxynonenal in dentate granule cells. Biol Pharm Bull 27:174-179.

Akerboom J, Carreras Calderón N, Tian L, Wabnig S, Prigge M, Tolö J, Gordus A, Orger MB, Severi KE, Macklin JJ, Patel R, Pulver SR, Wardill TJ, Fischer E, Schüler C, Chen TW, Sarkisyan KS, Marvin JS, Bargmann
CI, Kim DS, et al. (2013) Genetically encoded calcium indicators for multi-color neural activity imaging and combination with optogenetics. Front Mol Neurosci 6:2.

Ali F, Kwan AC (2020) Interpreting in vivo calcium signals from neuronal cell bodies, axons, and dendrites: a review. Neurophotonics 7:011402.

Alvarez J, Alvarez-Illera P, García-Casas P, Fonteriz RI, Montero M (2020) The role of $\mathrm{Ca}^{2+}$ signaling in aging and neurodegeneration: insights from Caenorhabditis elegans models. Cells 9:204.

Amberg GC, Earley S, Glapa SA (2010) Local regulation of arterial L-type calcium channels by reactive oxygen species. Circ Res 107:1002-1010.

Ashby MC, Daw MI, Issac JT (2008) AMPA receptors. In: The glutamate receptors (Gereau RW, Swanson GT, eds), pp 1-44. Totowa, NJ: Humana.

Back P, Braeckman BP, Matthijssens F (2012) ROS in aging Caenorhabditis elegans: damage or signaling? Oxid Med Cell Longev 2012:608478.

Barja G (2013) Updating the mitochondrial free radical theory of aging: an integrated view, key aspects, and confounding concepts. Antioxid Redox Signal 19:1420-1445.

Bayer KU, Schulman H (2019) CaM kinase: still inspiring at 40. Neuron 103:380-394.

Beckhauser TF, Francis-Oliveira J, De Pasquale R (2016) Reactive Oxygen Species: Physiological and Physiopathological Effects on Synaptic Plasticity J Exp Neurosci 10:23-48.

Ben Arous J, Tanizawa Y, Rabinowitch I, Chatenay D, Schafer WR (2010) Automated imaging of neuronal activity in freely behaving Caenorhabditis elegans. J Neurosci Methods 187:229-234.

Bienert GP, Schjoerring JK, Jahn TP (2006) Membrane transport of hydrogen peroxide. Biochim Biophys Acta 1758:994-1003.

Bindokas VP, Jordán J, Lee CC, Miller RJ (1996) Superoxide production in rat hippocampal neurons: selective imaging with hydroethidine. J Neurosci 16:1324-1336.

Bliss TV, Collingridge GL (1993) A synaptic model of memory: long-term potentiation in the hippocampus. Nature 361:31-39.

Boehm J, Kang MG, Johnson RC, Esteban J, Huganir RL, Malinow R (2006) Synaptic incorporation of AMPA receptors during LTP is controlled by a PKC phosphorylation site on GluR1. Neuron 51:213-225.

Bommel B, Konietzny A, Kobler O, Bär J, Mikhaylova M (2019) F-actin patches associated with glutamatergic synapses control positioning of dendritic lysosomes. EMBO J 38:e101183.

Braeckman BP, Smolders A, Back P, De Henau S (2016) In vivo detection of reactive oxygen species and redox status in Caenorhabditis elegans. Antioxid Redox Signal 25:577-592.

Brechet A, Buchert R, Schwenk J, Boudkkazi S, Zolles G, Siquier-Pernet K, Schaber I, Bildl W, Saadi A, Bole-Feysot C, Nitschke P, Reis A, Sticht H, Al-Sanna'a N, Rolfs A, Kulik A, Schulte U, Colleaux L, Abou Jamra R, Fakler B (2017) AMPA-receptor specific biogenesis complexes control synaptic transmission and intellectual ability. Nat Commun 8:15910.

Brenner S (2003) Nature's gift to science (Nobel lecture). Chembiochem 4:683-687.

Brini M, Cali T, Ottolini D, Carafoli E (2014) Neuronal calcium signaling: function and dysfunction. Cell Mol Life Sci 71:2787-2814.

Burbea M, Dreier L, Dittman JS, Grunwald ME, Kaplan JM (2002) Ubiquitin and AP180 regulate the abundance of GLR-1 glutamate receptors at postsynaptic elements in C. elegans. Neuron 35:107-120.

Catterall WA (2011) Voltage-gated calcium channels. Cold Spring Harb Perspect Biol 3:1-23.

Chaplin NL, Amberg GC (2012) Hydrogen peroxide mediates oxidant-dependent stimulation of arterial smooth muscle L-type calcium channels. Am J Physiol, Cell Physiol 302:C1382-C1393.

Choquet D, Triller A (2013) The dynamic synapse. Neuron 80:691-703.

Chronis N, Zimmer M, Bargmann CI (2007) Microfluidics for in vivo imaging of neuronal and behavioral activity in Caenorhabditis elegans. Nat Methods 4:727-731.

Cserne Szappanos H, Viola H, Hool LC (2017) L-type calcium channel: clarifying the "oxygen sensing hypothesis." Int J Biochem Cell Biol 86:32-36.

Dang AK, Chaplin NL, Murtazina DA, Boehm U, Clay CM, Amberg GC (2018) Subplasmalemmal hydrogen peroxide triggers calcium influx in gonadotropes. J Biol Chem 293:16028-16042.

Donato A, Kagias K, Zhang Y, Hilliard MA (2019) Neuronal sub-compartmentalization: a strategy to optimize neuronal function. Biol Rev Camb Philos Soc 94:1023-1037. 
Eales KL, Palygin O, O'Loughlin T, Rasooli-Nejad S, Gaestel M, Müller J, Collins DR, Pankratov Y, Corrêa SA (2014) The MK2/3 cascade regulates AMPAR trafficking and cognitive flexibility. Nat Commun 5:4701.

Ehlers MD (2000) Reinsertion or degradation of AMPA receptors determined by activity-dependent endocytic sorting. Neuron 28:511-525.

Esteves da Silva M, Adrian M, Schätzle P, Lipka J, Watanabe T, Cho S, Futai K, Wierenga CJ, Kapitein LC, Hoogenraad CC (2015) Positioning of AMPA receptor-containing endosomes regulates synapse architecture. Cell Rep 13:933-943.

Görlach A, Bertram K, Hudecova S, Krizanova O (2015) Calcium and ROS: a mutual interplay. Redox Biol 6:260-271.

Gray JM, Hill JJ, Bargmann CI (2005) A circuit for navigation in Caenorhabditis elegans. Proc Natl Acad Sci USA 102:3184-3191.

Griffin EF, Scopel SE, Stephen CA, Holzhauer AC, Vaji MA, Tuckey RA, Berkowitz LA, Caldwell KA, Caldwell GA (2019) ApoE-associated modulation of neuroprotection from $\mathrm{A} \beta$-mediated neurodegeneration in transgenic Caenorhabditis elegans. Dis Model Mech 12:dmm037218.

Grimm A, Eckert A (2017) Brain aging and neurodegeneration: from a mitochondrial point of view. J Neurochem 143:418-431.

Gordus A, Pokala N, Levy S, Flavell SW, Bargmann CI (2015) Feedback from network states generates variability in a probabilistic olfactory circuit. Cell 161:215-227.

Groc L, Bard L, Choquet D (2009) Surface trafficking of N-methyl-D-aspartate receptors: physiological and pathological perspectives. Neuroscience 158:4-18.

Guillaud L, Wong R, Hirokawa N (2008) Disruption of KIF17-Mint1 interaction by CaMKII-dependent phosphorylation: a molecular model of kinesin-cargo release. Nat Cell Biol 10:19-29.

Hall CN, Klein-Flugge MC, Howarth C, Attwell D (2012) Oxidative phosphorylation, not glycolysis, powers presynaptic and postsynaptic mechanisms underlying brain information processing. J Neurosci 32:89408951.

Halliwell B (1992) Reactive oxygen species and the central nervous system. J Neurochem 59:1609-1623.

Hangen E, Cordelières FP, Petersen JD, Choquet D, Coussen F (2018) Neuronal activity and intracellular calcium levels regulate intracellular transport of newly synthesized AMPAR. Cell Rep 24:1001-1012.e3.

Hanus C, Geptin H, Tushev G, Garg S, Alvarez-Castelao B, Sambandan S, Kochen L, Hafner AS, Langer JD, Schuman EM (2016) Unconventional secretory processing diversifies neuronal ion channel properties. Elife 5: e20609.

Hayashi Y, Shi SH, Esteban JA, Piccini A, Poncer JC, Malinow R (2000) Driving AMPA receptors into synapses by LTP and CaMKII: requirement for GluR1 and PDZ domain interaction. Science 287:2262-2267.

Hendricks M, Ha H, Maffey N, Zhang Y (2012) Compartmentalized calcium dynamics in a C. elegans interneuron encode head movement. Nature 487:99-103.

Henley JM, Wilkinson KA (2013) AMPA receptor trafficking and the mechanisms underlying synaptic plasticity and cognitive aging. Dialogues Clin Neurosci 15:11-27.

Henley JM, Wilkinson KA (2016) Synaptic AMPA receptor composition in development, plasticity and disease. Nat Rev Neurosci 17:337-350.

Hidalgo C, Arias-Cavieres A (2016) Calcium, reactive oxygen species, and synaptic plasticity. Physiology (Bethesda) 31:201-215.

Higley MJ, Sabatini BL (2012) Calcium signaling in dendritic spines. Cold Spring Harb Perspect Biol 4:a005686.

Hoerndli FJ, Maxfield DA, Brockie PJ, Mellem JE, Jensen E, Wang R, Madsen DM, Maricq AV (2013) Kinesin-1 regulates synaptic strength by mediating the delivery, removal, and redistribution of AMPA receptors. Neuron 80:1421-1437.

Hoerndli FJ, Wang R, Mellem JE, Kallarackal A, Brockie PJ, Thacker C, Madsen DM, Maricq AV (2015) Neuronal activity and CaMKII regulate kinesin-mediated transport of synaptic AMPARs. Neuron 86:457-474.

Huganir RL, Nicoll RA (2013) AMPARs and synaptic plasticity: the last 25 years. Neuron 80:704-717.

Ichinose S, Ogawa T, Hirokawa N (2015) Mechanism of activity-dependent cargo loading via the phosphorylation of KIF3A by PKA and CaMKIIa. Neuron 87:1022-1035.

Ju W, Morishita W, Tsui J, Gaietta G, Deerinck TJ, Adams SR, Garner CC, Tsien RY, Ellisman MH, Malenka RC (2004) Activity-dependent regulation of dendritic synthesis and trafficking of AMPA receptors. Nat Neurosci 7:244-253.
Kamsler A, Segal M (2003) Hydrogen peroxide modulation of synaptic plasticity. J Neurosci 23:269-276.

Kennedy MJ, Davison IG, Robinson CG, Ehlers MD (2010) Syntaxin-4 defines a domain for activity-dependent exocytosis in dendritic spines. Cell 141:524-535.

Kim CH, Lisman JE (2001) A labile component of AMPA receptor-mediated synaptic transmission is dependent on microtubule motors, actin and $\mathrm{N}$ ethylmaleimide-sensitive factor. J Neurosci 21:4188-4194.

Kim KW, Jin Y (2015) Neuronal responses to stress and injury in C. elegans. FEBS Lett 589:1644-1652.

Kim SK, Horvitz HR (1990) The Caenorhabditis elegans gene lin-10 is broadly expressed while required specifically for the determination of vulval cell fates. Genes Dev 4:357-371.

Kishida KT, Klann E (2007) Sources and targets of reactive oxygen species in synaptic plasticity and memory. Antioxid Redox Signal 9:233-244.

Klann E (1998) Cell-permeable scavengers of superoxide prevent long-term potentiation in hippocampal area CA1. J Neurophysiol 80:452-457.

Kwok TC, Ricker N, Fraser R, Chan AW, Burns A, Stanley EF, McCourt P, Cutler SR, Roy PJ (2006) A small-molecule screen in C. elegans yields a new calcium channel antagonist. Nature 441:91-95.

Laine V, Segor JR, Zhan H, Bessereau JL, Jospin M (2014) Hyperactivation of L-type voltage-gated $\mathrm{Ca}^{2+}$ channels in Caenorhabditis elegans striated muscle can result from point mutations in the IS6 or the IIIS4 segment of the 1 subunit. J Exp Biol 217:3805-3814.

Larsch J, Ventimiglia D, Bargmann CI, Albrecht DR (2013) High-throughput imaging of neuronal activity in Caenorhabditis elegans. Proc Natl Acad Sci USA 110:E4266-E4273.

Lee RY, Lobel L, Hengartner M, Horvitz HR, Avery L (1997) Mutations in the alpha 1 subunit of an L-type voltage-activated $\mathrm{Ca}^{2+}$ channel cause myotonia in Caenorhabditis elegans. EMBO J 16:6066-6076.

Liu Q, Kidd PB, Dobosiewicz M, Bargmann CI (2018) C. elegans AWA olfactory neurons fire calcium-mediated all-or-none action potentials. Cell 175:57-70.

Luo L, Wen Q, Ren J, Hendricks M, Gershow M, Qin Y, Greenwood J, Soucy ER, Klein M, Smith-Parker HK, Calvo AC, Colón-Ramos DA, Samuel AD, Zhang Y (2014) Dynamic encoding of perception, memory, and movement in a C. elegans chemotaxis circuit. Neuron 82:1115-1128.

Maricq AV, Peckol E, Driscoll M, Bargmann CI (1995) Mechanosensory signalling in C. elegans mediated by the GLR-1 glutamate receptor. Nature 378:78-81.

Massaad CA, Klann E (2011) Reactive oxygen species in the regulation of synaptic plasticity and memory. Antioxid Redox Signal 14:2013-2054.

Monteiro MI, Ahlawat S, Kowalski JR, Malkin E, Koushika SP, Juo P (2012) The kinesin-3 family motor KLP-4 regulates anterograde trafficking of GLR-1 glutamate receptors in the ventral nerve cord of Caenorhabditis elegans. Mol Biol Cell 23:3647-3662.

Neumann S, Chassefeyre R, Campbell GE, Encalada SE (2017) KymoAnalyzer: a software tool for the quantitative analysis of intracellular transport in neurons. Traffic 18:71-88.

Nimchinsky EA, Sabatini BL, Svoboda K (2002) Structure and function of dendritic spines. Annu Rev Physiol 64:313-353.

Park EC, Horvitz HR (1986) Mutations with dominant effects on the behavior and morphology of the nematode Caenorhabditis elegans. Genetics 113:821-852.

Park EC, Rongo C (2016) The p38 MAP kinase pathway modulates the hypoxia response and glutamate receptor trafficking in aging neurons. Elife 5:e12010.

Park EC, Glodowski DR, Rongo C (2009) The ubiquitin ligase RPM-1 and the p38 MAPK PMK-3 regulate AMPA receptor trafficking. PLoS One 4: e4284.

Petriv OI, Rachubinski RA (2004) Lack of peroxisomal catalase causes a progeric phenotype in Caenorhabditis elegans. J Biol Chem 279:1999620001.

Puthanveettil SV, Monje FJ, Miniaci MC, Choi YB, Karl KA, Khandros E, Gawinowicz MA, Sheetz MP, Kandel ER (2008) A new component in synaptic plasticity: upregulation of kinesin in the neurons of the gill-withdrawal reflex. Cell 135:960-973.

Reiner DJ, Newton EM, Tian H, Thomas JH (1999) Diverse behavioural defects caused by mutations in Caenorhabditis elegans unc-43 CaM kinase II. Nature 402:199-203. 
Ren SQ, Yan JZ, Zhang XY, Bu YF, Pan WW, Yao W, Tian T, Lu W (2013) $\mathrm{PKC} \lambda$ is critical in AMPA receptor phosphorylation and synaptic incorporation during LTP. EMBO J 32:1365-1380.

Rongo C, Kaplan JM (1999) CamKII regulates density of glutamatergic synapses in vivo. Nature 402:195-199.

Rosendale M, Jullié D, Choquet D, Perrais D, Wang Z, Edwards JG, Riley N, Provance DW, Karcher R, Li X, Davison IG, Ikebe M, Mercer JA, Kauer JA, Ehlers MD, Cullen PJ (2003) Spatial and temporal regulation of receptor endocytosis in neuronal dendrites revealed by imaging of single vesicle formation. Curr Biol 135:1840-1847.

Sabatini BL, Maravall M, Svoboda K (2001) $\mathrm{Ca}^{2+}$ signaling in dendritic spines. Curr Opin Neurobiol 11:349-356.

Sarasija S, Norman KR (2015) A $\gamma$-secretase independent role for presenilin in calcium homeostasis impacts mitochondrial function and morphology in Caenorhabditis elegans. Genetics 201:1453-1466.

Sarasija S, Laboy JT, Ashkavand Z, Bonner J, Tang Y, Norman KR (2018) Presenilin mutations deregulate mitochondrial $\mathrm{Ca}^{2+}$ homeostasis and metabolic activity causing neurodegeneration in Caenorhabditis elegans. Elife 7:e33052.

Scragg JL, Dallas ML, Wilkinson JA, Varadi G, Peers C (2008) Carbon monoxide inhibits L-type $\mathrm{Ca}^{2+}$ channels via redox modulation of key cysteine residues by mitochondrial reactive oxygen species. J Biol Chem 283:24412-24419.

Sengupta P, Samuel A (2009) C. elegans: a model system for systems neuroscience. Curr Opin Neurobiol 19:637-643.

Serrano-Saiz E, Poole RJ, Felton T, Zhang F, De La Cruz ED, Hobert O (2013) XModular control of glutamatergic neuronal identity in C. elegans by distinct homeodomain proteins. Cell 155:659-673.

Setou M, Seog DH, Tanaka Y, Kanai Y, Takei Y, Kawagishi M, Hirokawa N (2002) Glutamate-receptor-interacting protein GRIP1 directly steers kinesin to dendrites. Nature 417:83-87.

Sies H (2017) Hydrogen peroxide as a central redox signaling molecule in physiological oxidative stress: oxidative eustress. Redox Biol 11:613619.

Spiró Z, Arslan MA, Somogyvári M, Nguyen MT, Smolders A, Dancsó B, Németh N, Elek Z, Braeckman BP, Csermely P, So'ti C (2012) RNA interference links oxidative stress to the inhibition of heat stress adaptation. Antioxid Redox Signal 17:890-901.

Stefanatos R, Sanz A (2018) The role of mitochondrial ROS in the aging brain. FEBS Lett 592:743-758

Stiernagle T (2006) Maintenance of C. elegans. WormBook 1-11.

Stucchi R, Plucińska G, Hummel JJ, Zahavi EE, Guerra San Juan I, Klykov O, Scheltema RA, Altelaar AF, Hoogenraad CC (2018) Regulation of KIF1A-driven dense core vesicle transport: $\mathrm{Ca}^{2+} / \mathrm{CaM}$ controls DCV binding and liprin- $\alpha /$ TANC2 recruits DCVs to postsynaptic sites. Cell Rep 24:685-700.

Tang S, Yasuda R (2017) Imaging ERK and PKA activation in single dendritic spines during structural plasticity. Neuron 93:1315-1324.e3.
Tardiff DF, Jui NT, Khurana V, Tambe MA, Thompson ML, Chung CY, Kamadurai HB, Kim HT, Lancaster AK, Caldwell KA, Caldwell GA, Rochet JC, Buchwald SL, Lindquist S (2013) Yeast reveal a "druggable" Rsp5/Nedd4 network that ameliorates $\alpha$-Synuclein toxicity in neurons. Science 342:979-983.

Thiels E, Urban NN, Gonzalez-Burgos GR, Kanterewicz BI, Barrionuevo G, Chu CT, Oury TD, Klann E (2000) Impairment of long-term potentiation and associative memory in mice that overexpress extracellular superoxide dismutase. J Neurosci 20:7631-7639.

Todorovic SM, Jevtovic-Todorovic V (2014) Redox regulation of neuronal voltage-gated calcium channels. Antioxid Redox Signal 21:880-891.

Trent C, Tsuing N, Horvitz HR (1983) Egg-laying defective mutants of the nematode Caenorhabditis elegans. Genetics 104:619-647.

Treusch S, Hamamichi S, Goodman JL, Matlack KE, Chung CY, Baru V, Shulman JM, Parrado A, Bevis BJ, Valastyan JS, Han H, LindhagenPersson M, Reiman EM, Evans DA, Bennett DA, Olofsson A, DeJager PL, Tanzi RE, Caldwell KA, Caldwell GA, et al. (2011) Functional links between $\mathrm{A} \beta$ toxicity, endocytic trafficking, and Alzheimer's disease risk factors in yeast. Science 334:1241-1245.

Umemura T, Rapp P, Rongo C (2005) The role of regulatory domain interactions in UNC-43 CaMKII localization and trafficking. J Cell Sci 118:3327-3338.

Wilson C, González-Billault C (2015) Regulation of cytoskeletal dynamics by redox signaling and oxidative stress: implications for neuronal development and trafficking. Front Cell Neurosci 9:381.

Wilson C, Muñoz-Palma E, González-Billault C (2018) From birth to death: a role for reactive oxygen species in neuronal development. Semin Cell Dev Biol 80:43-49.

Yermolaieva O, Brot N, Weissbach H, Heinemann SH, Hoshi T (2000) Reactive oxygen species and nitric oxide mediate plasticity of neuronal calcium signaling. Proc Natl Acad Sci USA 97:448-453.

Yudowski GA, Puthenveedu MA, Leonoudakis D, Panicker S, Thorn KS, Beattie EC, Von Zastrow M (2007) Real-time imaging of discrete exocytic events mediating surface delivery of AMPA receptors. J Neurosci 27:11112-11121.

Zheng Y, Brockie PJ, Mellem JE, Madsen DM, Maricq AV (1999) Neuronal control of locomotion in C. elegans is modified by a dominant mutation in the GLR-1 ionotropic glutamate receptor. Neuron 24:347-361.

Zheng Z, Keifer J (2008) Protein kinase C-dependent and independent signaling pathways regulate synaptic GluR1 and GluR4 AMPAR subunits during in vitro classical conditioning. Neuroscience 156:872-884.

Zullo JM, Drake D, Aron L, O'Hern P, Dhamne SC, Davidsohn N, Mao CA, Klein WH, Rotenberg A, Bennett DA, Church GM, Colaiácovo MP, Yankner BA (2019) Regulation of lifespan by neural excitation and REST. Nature 574:359-364. 